\title{
The first quarter of the C-terminal domain of Abelson regulates the WAVE regulatory complex and Enabled in axon guidance
}

\author{
Han Sian Joshua Cheong, Mark Nona, Samantha Barbara Guerra and Mark Francis VanBerkum *
}

\begin{abstract}
Background: Abelson tyrosine kinase (Abl) plays a key role in axon guidance in linking guidance receptors to actin dynamics. The long C-terminal domain (CTD) of Drosophila Abl is important for this role, and previous work identified the 'first quarter' (1Q) of the CTD as essential. Here, we link the physical interactions of $1 \mathrm{Q}$ binding partners to Abl's function in axon guidance.

Methods: Protein binding partners of $1 \mathrm{Q}$ were identified by GST pulldown and mass spectrometry, and validated using axon guidance assays in the embryonic nerve cord and motoneurons. The role of $1 \mathrm{Q}$ was assessed genetically, utilizing a battery of $\mathrm{Ab} /$ transgenes in combination with mutation or overexpression of the genes of pulled down proteins, and their partners in actin dynamics. The set of $A b /$ transgenes had the following regions deleted: all of 1Q, each half of $1 \mathrm{Q}$ ('eighths', $1 \mathrm{E}$ and 2E) or a PxxP motif in 2E, which may bind SH3 domains.

Results: GST pulldown identified Hem and Sra-1 as binding partners of 1Q, and our genetic analyses show that both proteins function with Abl in axon guidance, with Sra-1 likely interacting with 1Q. As Hem and Sra-1 are part of the actin-polymerizing WAVE regulatory complex (WRC), we extended our analyses to Abi and Trio, which interact with Abl and WRC members. Overall, the $1 \mathrm{Q}$ region (and especially $2 \mathrm{E}$ and its PxxP motif) are important for Abl's ability to work with WRC in axon guidance. These areas are also important for Abl's ability to function with the actin regulator Enabled. In comparison, $1 E$ contributes to Abl function with the WRC at the midline, but less so with Enabled.

Conclusions: The $1 \mathrm{Q}$ region, and especially the $2 \mathrm{E}$ region with its PxxP motif, links Abl with the WRC, its regulators Trio and $\mathrm{Abi}$, and the actin regulator Ena. Removing $1 \mathrm{E}$ has specific effects suggesting it may help modulate Abl's interaction with the WRC or Ena. Thus, the $1 \mathrm{Q}$ region of Abl plays a key role in regulating actin dynamics during axon guidance.
\end{abstract}

\section{Introduction}

Abelson tyrosine kinase (Abl) is a key signaling molecule involved in a plethora of cell processes [1-4]. Arguably, it might be best known as a key regulator of actin dynamics during development of the organism, including cell morphogenesis, migration, and axon guidance in both vertebrate and invertebrate model systems [1-3]. Here, we focus on the function of the sole $A b l$ homolog in Drosophila during development of axon tracts. Loss

\footnotetext{
* Correspondence: mvb@wayne.edu

Department of Biological Sciences, Wayne State University, Detroit, MI 48202, USA
}

of maternal and zygotic $\mathrm{Abl}$ in the embryo results in disruption of epithelial morphogenesis, and also severely impacts commissure formation in the nerve cord $[5,6]$. However, if maternal loading is retained, zygotic loss of $A b l$ results in more specific axon guidance defects, revealing key roles for $\mathrm{Abl}$ as a link between upstream guidance receptors and the cytoskeletal elements underlying growth cone extension and steering.

In the embryonic nerve cord, zygotic loss of $A b l$ causes aberrant crossing of axons at the midline [7-10], a simple phenotype that has been helpful in genetic analyses linking $\mathrm{Abl}$ activity to the transduction of both midline attractive

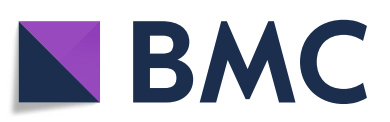

(C) The Author(s). 2020 Open Access This article is licensed under a Creative Commons Attribution 4.0 International License, which permits use, sharing, adaptation, distribution and reproduction in any medium or format, as long as you give appropriate credit to the original author(s) and the source, provide a link to the Creative Commons licence, and indicate if changes were made. The images or other third party material in this article are included in the article's Creative Commons licence, unless indicated otherwise in a credit line to the material. If material is not included in the article's Creative Commons licence and your intended use is not permitted by statutory regulation or exceeds the permitted use, you will need to obtain permission directly from the copyright holder. To view a copy of this licence, visit http://creativecommons.org/licenses/by/4.0/ The Creative Commons Public Domain Dedication waiver (http://creativecommons.org/publicdomain/zero/1.0/) applies to the data made available in this article, unless otherwise stated in a credit line to the data. 
(Frazzled) $[8,11,12]$ and repulsive (Roundabout) $[10,13$, $14]$ cues. $A b l$ and Dscam 1 also genetically interact in dendritic arborization [15]. In addition, loss of Abl signaling impairs motoneuron axon guidance in the intersegmental nerve b (ISNb) and segmental nerve a (SNa) $[16,17]$. In modulating these axon guidance events, Abl cooperates with many partners known to be important in regulating cytoskeletal dynamics, such as the actin-regulating proteins Enabled (ena) [18, 19], Abelson-interacting protein (Abi) [20] and Chickadee/profilin [16], the adaptor protein Disabled [17], and the Rac guanine exchange factor (GEF) Trio [21]; even the microtubule-associated protein Orbit may be a target [14]. Indeed, Abl is thought to play a key role in balancing between linear and branched actin polymerization that underlie growth cone dynamics [21]. However, a well-defined, complete Abl signaling pathway remains elusive, due to the large number of Abl partners and likely interplay between them.

Initial work focused on the identification of Abl phosphorylation targets, most notably Roundabout, Frazzled, Ena, Abi and Trio [8, 13, 22, 23], although in some cases, the significance of phosphorylation remains in question (e.g. see [22]). Moreover, early experiments in Drosophila also demonstrate that a kinase inactive-version of $\mathrm{Abl}$ retains significant function, even rescuing lethality of $A b l$ loss [24], although partial loss of function is demonstrated in developmental events in maternal-zygotic $\mathrm{Abl}$ mutant embryos [5]. Thus, even without kinase activity, $\mathrm{Abl}$ is still a key player in regulating actin dynamics, yet how Abl does so remains in question. To further develop our understanding of Abl signaling, we assessed the contribution of other regions of Abl to its function.

Early work demonstrated that the long C-terminal domain (CTD) of Abl is critical to its function, as the CTD of murine Abl1 cannot substitute for the Drosophila Abl CTD in vivo [24]. The CTD of Drosophila Abl spans $\sim 1100$ amino acids and contains no known domains except for a small F-actin binding domain (Fig. 2a). In addition, two putative EVH1-binding motifs (F/LPPPP) were initially thought to recruit Ena [25]. Yet both regions can be removed without unduly affecting Abl function in flies $[5,11]$. Homology-based methods comparing insect Abl homologs alone identified 4 small conserved regions of the CTD, but 3 of the 4 can be removed with no apparent effect [5]. The sole important region ('conserved region 1', or CR1) contains a PxxP motif that may be functionally relevant [5], as discussed below.

On the other hand, the Abl CTD is characterized by the presence of intrinsic disorder spanning most of its sequence [26]. Intrinsically disordered regions lack welldefined secondary or tertiary structure, and are flexible and structurally heterogenous [27]. Yet, the role of intrinsically disordered regions in cell signaling is increasingly appreciated, due to their potential ability to bind multiple protein partners, as well as enrichment in posttranslational modification sites [28]. Furthermore, disordered regions appear to have faster evolutionary rates $[29,30]$, perhaps explaining the limited homology between vertebrate and invertebrate species, as well as the limited success of homology-based approaches to understanding Abl CTD function. We reasoned that the long $\mathrm{CTD}$ of $\mathrm{Abl}$ is a prime candidate for a scaffolding function, thus accounting for its critical importance in Drosophila Abl function.

Our earlier study tested this hypothesis by systematically analyzing the contribution of the CTD region to $\mathrm{Abl}$ function in viability and axon guidance [26]. The CTD was somewhat arbitrarily divided into four large regions (we called them 'quarters', or $1 \mathrm{Q}-4 \mathrm{Q}$ ), taking care not to disrupt the few known peptide motifs. These regions were then either deleted from full-length Abl or added onto the Abl N-terminus alone, and the resulting transgenes were tested in axon guidance. Surprisingly, this analysis revealed that much of the CTD could be removed without significantly altering Abl function in axon guidance or viability. Indeed, only one region, 1Q, was identified as absolutely necessary for Abl function, although another region, the third quarter, aided in localizing $\mathrm{Abl}$ to axons, allowing a small transgene carrying only $1 \mathrm{Q}$ and $3 \mathrm{Q}$ with the $\mathrm{N}$-terminus (AblN-1Q-3Q) to behave close to a wild-type Abl.

Unfortunately, few sequence motifs may be found within the 1Q region. The second half of $1 \mathrm{Q}$ is similar to the CR1 region which plays a role in actin based morphogenetic events [5] and is slightly more conserved within insects. This region also harbors a small PxxP motif that may mediate physical interactions with $\mathrm{SH} 3$ domains, which are widely distributed among proteins in nature $[31,32]$. In vertebrates, three such motifs mediate interactions with the SH3 domains of Abi, Crk, Nck, and Grb [33-38]. Thus, these proteins are potential candidates for binding to 1Q in Drosophila, although we note that the conservation of the surrounding amino acids, which often contribute to binding [39], are much poorer [26]. To dissect these regions, we divided the $1 \mathrm{Q}$ region into halves, calling them the first and second eighths (1E and $2 \mathrm{E})$, and also created a third mutant removing only the four amino acids of the PxxP motif. Deletion of either 2E or the PxxP motif alone significantly inhibits Abl function in axon guidance $[5,26]$. However, deletion of the entire $1 \mathrm{Q}$ sequence impacts $\mathrm{Abl}$ function even more, suggesting the presence of other unknown regions of interest [26].

Here, we continue to investigate why the $1 \mathrm{Q}$ region is critical to Abl function in axon guidance. First, we screened for protein binding partners of 1Q by GST pulldown, and identified Hematopoietic protein (Hem) and Specifically Rac1-associated protein 1 (Sra-1). Both proteins are part of the WAVE regulatory complex (WRC), a 5-subunit 
complex that is activated by Rac GTPases to nucleate branched actin polymerization [40-43]. Interestingly, the WRC component Abi, as well as the Rac GEF Trio, are known to genetically interact with Abl during axon guidance $[8,20,21]$. As such, we elected to continue genetic analysis of the Abl-Hem-Sra-1 interaction to determine if the $1 \mathrm{Q}$ region regulates the WRC through these other $\mathrm{Abl}$ partners. Genetic analyses indicate that $1 \mathrm{Q}$, and especially 2E and its PxxP motif, does indeed work with the WRC in axon guidance, as mutations of these regions genetically interacts with $A b i$ and trio. It has been proposed that the WRC acts in parallel to an Ena-dependent pathway [21], yet as an unanticipated outcome, the 1Q region is also important for Abl's ability to regulate Ena despite the lack of any Ena-binding motifs in 1Q. Clearly, the $1 \mathrm{Q}$ region and especially $2 \mathrm{E}$ and its PxxP motif, connects Abl to multiple players of actin dynamics. On the other hand, the $1 \mathrm{E}$ region may be involved in fine-tuning Abl function with the WRC and Ena. We suggest that 1Q functions at the intersection between linear and branched actin polymerization, and aids $\mathrm{Abl}$ in linking receptors to modulate this balance during axon guidance.

\section{Methods}

\section{Recombinant GST protein expression and purification}

The Abl 1Q region was amplified from a pMT-Abl vector as an AgeI/NotI fragment and cloned into a modified pGEX-6p1 vector that adds a C-terminal FLAG tag. The forward and reverse primers used are TTG AAC CGG TCA TGC GCT GGA GCA CAT GTT T and GTG AGC GGC CGC GTC CAT TCG TGC TGA GGT CGT C (italicized regions are complementary to $1 \mathrm{Q}$ ). Transgenic GST and GST-1Q were expressed in BL21 E. coli at $18^{\circ} \mathrm{C}$ for 2 days in autoinduction medium [44]. E. coli was pelleted and lysed using B-PER reagent (Thermo Fisher, Waltham, MA, USA), and purified on Glutathione Sepharose 4B beads (GE Healthcare, Chicago, IL, USA). Following elution using $10 \mathrm{mM}$ reduced glutathione $+1 \%$ Nonidet $\mathrm{P}-40$, eluates were dialyzed against dialysis buffer $(1 \%$ Nonidet P-40, $25 \mathrm{mM}$ Tris pH 7.4, $150 \mathrm{mM} \mathrm{NaCl}$ ). Protein concentrations were determined using Advanced Protein Assay reagent (Cytoskeleton, Inc., Denver, CO, USA).

\section{GST pulldown}

Roughly $4 \mathrm{ml}$ of frozen overnight collections of embryos were lysed in $20 \mathrm{~mL}$ total volume of lysis buffer $(0.25 \%$ IGEPAL CA-630, $25 \mathrm{mM}$ Tris pH 7.4, $100 \mathrm{mM} \mathrm{NaCl}, 5 \%$ glycerol, $1 \mathrm{mM} \mathrm{MgCl}, 1 \mathrm{mM} \mathrm{CaCl}, 10 \mathrm{mM} \mathrm{NaF}, 2 \mathrm{mM}$ $\mathrm{Na}_{3} \mathrm{VO}_{4}$ pH 10, $1 \mathrm{mM}$ ATP, 1x Problock Gold protease inhibitor cocktail (GoldBio, St Louis, MO, USA) by homogenization in a $15 \mathrm{~mL}$ Dounce homogenizer (10 strokes of each pestle), then sonicated for 1 min total sonication time with $5 \mathrm{~s}$ per pulse and 10s breaks at amplitude 5 with a Misonix S-4000 Sonicator (Qsonica, Newtown, CT,
USA) with a $1 / 16$ " probe. Debris was pelleted by centrifugation for $10 \mathrm{~min}$ at $7.2 \mathrm{k}$ xG. Lysate was consecutively precleared for $20 \mathrm{~min}$ in $2 \mathrm{ml}, 1 \mathrm{ml}$, and $0.5 \mathrm{~mL}$ of Glutathione Sepharose 4B beads (GE Healthcare, Chicago, IL, USA).

For pulldown, $75 \mathrm{uL}$ Glutathione Sepharose 4B beads were loaded with $200 \mu \mathrm{g}$ bait protein (GST, GST-1Q). Beads were incubated with $3 \mathrm{~mL}$ of lysate for $1.5 \mathrm{~h}$ at $4{ }^{\circ} \mathrm{C}$, then washed 5 times with $500 \mathrm{uL}$ lysis buffer. Copurified proteins were eluted in $3 \times 33 \mathrm{uL} 50 \mathrm{mM}$ glutathione in lysis buffer (+ 0.3 M Tris $\mathrm{pH}$ 7.4), and fractions were combined. All experiments were performed in triplicate, with separate preparations of embryo lysate.

In preparation for mass spectrometry, polyacrylamide gel electrophoresis was carried out with either MiniPROTEAN TGX 4-20\% precast gels (Bio-Rad, Hercules, CA, USA) or hand-cast gels with a neutral-pH gel buffer (0.1 M tris- $\mathrm{AcOH} \mathrm{pH} 7,0.36 \mathrm{M}$ Glycine). Gels were run with a running buffer of $25 \mathrm{mM}$ tris, $192 \mathrm{mM}$ glycine, $0.1 \%$ SDS. Whole protein staining with Sypro Ruby stain (Thermo Fisher, Waltham, MA, USA) was carried out as per manufacturer instructions, and regions of interest (matched areas between GST and GST-1Q, above and below bait bands) were excised for mass spectrometry.

\section{Mass spectrometry and analysis}

32 gel slices were submitted to the Wayne State University Proteomics Core. The gel pieces were first washed with water and $25 \mathrm{mM} \mathrm{NH}_{4} \mathrm{HCO}_{3}, 50 \% \mathrm{ACN}$ for $15 \mathrm{~min}$ each. The liquid was removed, and the gel pieces were dehydrated in $100 \% \mathrm{ACN}$ for $5 \mathrm{~min}$, rehydrated in 50 $\mathrm{mM} \mathrm{NH} \mathrm{NHCO}_{3}$, followed by addition of an equal volume of $100 \%$ ACN. After incubation for $15 \mathrm{~min}$, all liquid was removed and the gel pieces dehydrated once again in $100 \% \mathrm{ACN}$ for $5 \mathrm{~min}$, and vacuum-desiccated for $5 \mathrm{~min}$ in a speed vac. The following was then performed: reduction with $5 \mathrm{mM}$ DTT, $50 \mathrm{mM} \mathrm{NH}_{4} \mathrm{HCO}_{3}$;

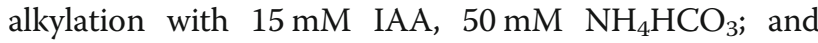
overnight digestion with sequencing-grade trypsin (Promega, Madison, WI, USA) in $40 \mathrm{mM} \mathrm{NH} \mathrm{NCO}_{3}, 0.01 \%$ Protease Max (Promega, Madison, WI, USA), and $1 \mathrm{mM}$ $\mathrm{CaCl}_{2}$. Following digestion, peptides were extracted from the gel plugs using $0.5 \%$ TFA, desiccated and solubilized in $0.1 \%$ FA.

The peptides were separated by reversed-phase chromatography with Acclaim PepMap100 C18 columns (Thermo Fisher, Waltham, MA, USA), followed by ionization with the Nanospray Flex Ion Source (Thermo Fisher, Waltham, MA, USA), and introduced into a QExactive versus Fusion mass spectrometer (Thermo Fisher, Waltham, MA, USA).

Abundant species were fragmented with high energy collision-induced dissociation (HCID for QEx) or collision-induced dissociation (CID for Fusion). Data analysis was performed using Proteome Discoverer 1.4 
(Thermo Fisher, Waltham, MA, USA) which incorporated the Mascot (Matrix Science, Boston, MA, USA) and Sequest algorithms (Thermo Fisher, Waltham, MA, USA). The Uniprot_Dros_Compl_20160407 database was searched for Drosophila protein sequences and a reverse decoy protein database was run simultaneously for false discovery rate (FDR) determination. Secondary analysis was performed using Scaffold 4.5.3 (Proteome Software, Portland, OR, USA). Minimum protein identification probability was set at $<=1-2 \%$ FDR with 2 unique peptides at $<=1 \%$ FDR minimum peptide identification probability.

Mascot, Sequest, and X! Tandem were searched with a fragment ion mass tolerance of $0.02 / 0.05 \mathrm{Da}$ and a parent ion tolerance of 10/20 PPM. Carbamidomethylation of cysteine was specified in Mascot, Sequest, and X! Tandem as a fixed modification. Deamidation of asparagine and glutamine, oxidation of methionine, and acetylation of the Nterminus were specified in Mascot \& Sequest as variable modifications. Glu- $>$ pyro-Glu of the n-terminus, ammonia-loss of the N-terminus, gln- > pyro-Glu of the Nterminus, deamidation of asparagine and glutamine, oxidation of methionine, and acetylation of the $\mathrm{N}$-terminus were specified in X! Tandem as variable modifications.

\section{Fly genetics and stocks}

Drosophila melanogaster were cultured at $25^{\circ} \mathrm{C}$ on standard cornmeal-molasses medium on a $12 \mathrm{~h}$ light/ dark cycle. The following alleles, deficiencies and drivers were used: $A b l^{4}, A b l^{2}$, trio $^{1}, A b i^{K O}, \mathrm{Hem}^{I 4-48}$ ena ${ }^{23}$,

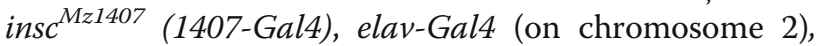
and $D f(3 R)$ Exel6174 $\left(\mathrm{Sra}^{-1}{ }^{D f}\right)$. UAS-Abl transgene stocks are as described below. In addition, the following UAS transgenes were also used: UAS-trio. B (on chromosome 3) [45], UAS-ena. His6 (on chromosome 3), UAS-mCherry.Abi (on chromosome 2 at ZH-51D attP landing site),

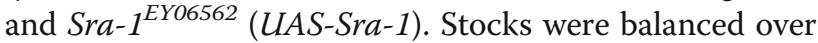
LacZ-containing balancer chromosomes (TM3 Sb actlacZ, CyO elav-lacZ, TM6 ase-lacZ or TM6 T8-lacZ) where appropriate. $e n a^{23}$ stocks were instead balanced over the nearby betaTub56 ${ }^{k 00705}$ LacZ enhancer trap due to stock health reasons. The trio ${ }^{1}, A b i^{K O}$, and UAS-trio122 stocks were a kind gift from Dr. Edward Giniger (NINDS). Other fly stocks were obtained from Bloomington Drosophila Stock Center or were pre-existing in our lab.

\section{Transgenic constructs}

UAS-Abl transgenes that are wild-type or carry the $\Delta 1 \mathrm{Q}, \Delta 2 \mathrm{E}$ or $\Delta \mathrm{P}$ deletions are as previously described [26]. The Abl $\Delta 1 \mathrm{E}$ transgene was made by site-directed mutagenesis of a PUASTattB-Abl plasmid using the following primers (regions complementary to Abl italicized): AGG AGA CCG GTC TCA CGC CGA ACG $C C C A C$ and GTG AGA CCG GTC TCC TGC TTT TCC ACC GCT TCG G. The PCR product was digested with AgeI and self-ligated. All Abl transgenes used here were inserted into the $\mathrm{ZH}$-attP-22A landing via Phi C-31 transgenesis [46]. All fly germline transformations were carried out by Rainbow Transgenic Flies, Inc.

\section{Immunoblotting}

To determine transgenic expression levels, $A b l$ transgenes were expressed in third instar larvae with 1407Gal4 at $25^{\circ} \mathrm{C}$. Separately, a UAS-RFP-FLAG transgene was expressed with 1407 -Gal4 and elav-Gal4 at $18^{\circ} \mathrm{C}$ and $25^{\circ} \mathrm{C}$. For both experiments, 5 nerve cords per genotype were dissected and lysed in $200 \mu \mathrm{L}$ SDS sample buffer, ran on SDS-PAGE gels, and transferred to PVDF membranes. Blots were incubated with 1:10000 Rat antiFLAG L5 (Thermo Fisher, Cat\# MA1-142-1MG, Waltham, MA, USA) and 1:500 Mouse anti-beta-tubulin E7 (DSHB, Cat\# E7-s, Iowa City, IA, USA) in $0.5 \%$ nonfat milk in PBS-Tween (1x PBS, $0.1 \%$ Tween-20). After washing, blots were incubated with 1:20000 HRPconjugated Goat anti-rat antibody (Jackson Immunoresearch, Cat\# 112-035-003, West Grove, PA, USA) and 1:20000 HRP-conjugated goat anti-mouse antibody (Jackson Immunotech, Cat\# 115-035-003, West Grove, PA, USA) in $0.5 \%$ nonfat milk in PBS-Tween. Chemiluminescent detection was carried out with Clarity Western ECL Substrate (Bio-Rad, Hercules, CA, USA). Dissections and western blots were repeated 3 times.

\section{Fly mating schemes}

Crosses for ISNb bypass with endogenous Abl present use the same basic scheme, where the female carries the Gal4 driver (1407-Gal4 or elav-Gal4) and any loss-offunction alleles balanced against a LacZ balancer, while the male carries all $A b l$ transgenes and any other transgenes used. Crosses in $A b l^{4 / 2}$ mutants were carried out with a similar scheme, where the female carries the Gal4 driver, $A b l^{4}$ allele and any other loss-of-function alleles, while the male carries all transgenes and the $A b l^{2}$ allele. However, as we carried out these mating schemes, we observed that a small proportion of embryos that were heterozygous for either Hem, Sra-1 or trio (without inheriting any balancers), but had a female parent with the TM6 balancer, had unexplained, highly-penetrant bypass phenotypes. This observation is similar to a previously observed TM6 balancer-induced maternal effect [47]. This effect was not observed when only the male parent carried the TM6 balancer. Thus, we reversed the parental sexes of all crosses that require TM6 balancers $\left(\mathrm{Sra}-1^{D f}\right.$ and $\mathrm{Hem}^{j 4-48}$ for the bypass assay, and Sra-1 ${ }^{\mathrm{Df}}$ and trio $^{1}$ for $A b l^{4 / 2}$ embryos).

\section{Embryo collection and immunohistochemistry}

Embryos were collected by standard techniques as previously described [7]. Flies were allowed to deposit 
embryos overnight on apple juice agar in mini embryo collection cages (Genesee Scientific, San Diego, CA, USA). Collected embryos were dechorionated with $25 \%$ bleach, fixed in formaldehyde-saturated heptane for 45 min with gentle rocking. Vitelline membranes were cracked by vigorous shaking in methanol, followed by immediate rehydration in PBT (1x PBS, 0.1\% Triton X100). To mark embryos carrying LacZ transgenes, embryos were then incubated in X-gal staining solution ( $1 \mathrm{x}$ PBS, $2 \mathrm{mM} \mathrm{MgCl} 2,5 \mathrm{mM}$ potassium ferrocyanide, $5 \mathrm{mM}$ potassium ferricyanide, $8 \mu \mathrm{LL}^{-1} 5 \% \mathrm{X}$-gal in dimethylformamide) for 1-12 h. Embryos were post-fixed in $4 \%$ formaldehyde in PBS for 10 min.

Embryos were incubated with mouse monoclonal antibody 1D4 supernatant (DSHB, Cat\# 1D4 anti-Fas II-s, Iowa City, IA, USA) at 1:10 dilution, or concentrate (DSHB, Cat\# 1D4 anti-Fas II-c, Iowa City, IA, USA) at 1: 100 dilution in PBT $+0.5 \%$ nonfat milk overnight. Following washing, embryos were incubated with polyclonal HRP-conjugated goat anti-mouse antibody (Jackson Immunotech, Cat\# 115-035-003, West Grove, PA, USA) for $4 \mathrm{~h}$. Chromogenic detection was carried out by incubation of embryos in Stable DAB solution (Thermo Fisher, Waltham, MA, USA). Embryos were then washed with PBT and cleared in 70\% glycerol. Imaging was carried out on a Leica DM5500B (Leica Microsystems, Buffalo Grove, IL, USA) microscope with differential interference contrast optics and an HCX PL APO 100x/1.40-0.70 OIL objective. Images were captured with a Leica DFC425C color camera using the LAS AF acquisition software.

\section{Embryonic phenotype quantification}

Embryos were sorted by developmental stages as previously defined [48], and were counted by a trained scorer blinded to embryo genotypes. Scoring was done on a Leica DM5500B microscope (Leica Microsystems, Buffalo Grove, IL, USA) at 1000x magnification with brightfield and differential interference contrast optics. For midline crossover defects, whole mount embryos of stages 16-17 were analyzed. All abdominal and thoracic segments were evaluated for each embryo. Ectopic crossing overs were defined as Fas2-positive axon bundles crossing the midline and joining the Fas2-positive fascicles on the opposite side. Three replicates per genotype and $\sim 30$ embryos per replicate were counted. Data were pooled for experiments that used the same control crosses. No estimation of required sample size based on statistical power was carried out.

For ISNb defects, only stage 17 embryos were counted, and only abdominal segments $2-7$ were evaluated as these have identical ISNb projections. For the bypass phenotype, full bypass was defined as complete failure of the ISNb to de-fasciculate from the ISN, with no visible axons leaving at the normal branch point. Partial bypass was defined as segments with visibly thinner ISNb branches, branching at a later point than expected, or branching followed by rejoining to the ISN. For the stop short phenotype, the last position of successful innervation was recorded per hemisegment, which may be at any of the muscle clefts $12 / 13$ (wild-type), 6/13 or 6/7. Hemisegments with ISNb bypass could not be evaluated for stop shorts, and thus were excluded from stop short analysis. A variable number of hemisegments were counted per embryo due to variations in embryo positioning and occasional damage. As such, the n-value represents number of hemisegments, as previously defined [16]. For each experiment, three replicates per genotype and 60-100 hemisegments over $\sim 10 \mathrm{em}$ bryos per replicate were counted. Data were pooled for experiments that used the same control crosses. No estimation of required sample size based on statistical power was carried out.

\section{Statistical analyses}

For mass spectrometry data, statistical analysis was carried out using Scaffold Viewer version 4.4.8 (Proteome Software, Portland, OR, USA). Spectral counts for each protein were compared between 3 replicates of GST-1Q and GST by pairwise t-tests with Hochberg-Benjamini correction at a threshold of $p \leq 0.05$.

All other statistical analyses were carried out in $\mathrm{R}$ version 3.6.1 (R Foundation, Vienna, Austria) with the multcomp [49], MASS [50], ggplot2 [51], car [52], DHARMa (http:// florianhartig.github.io/DHARMa/), and emmeans (https:// github.com/rvlenth/emmeans) packages. For counts of midline crossing over defects in $A b l^{4 / 2}$ embryos, data were pooled for all datasets that use the same control crosses, and fitted to a negative binomial generalized linear model with crossovers per embryo as the response variable, and $A b l$ transgene and other genotype (manipulation of $A b l$ pathway genes) as the two predictor variables. The dispersion test [53-55] was used to determine overdispersion. Diagnostics were carried out with scaled residual plots using the DHARMa package. Post-hoc analysis was carried out by comparisons of the estimated marginal means [56] with the emmeans package, at a threshold of $p \leq 0.05$ with the Holm method of $p$-value adjustment. A set of preplanned treatment-vs-control comparisons were made as follows: for each $\mathrm{Abl}$ transgene, crossing overs were compared between the $A b l^{4 / 2}$ condition alone and other genotypes (manipulation of $A b l$ pathway genes). Furthermore, within each genotype ('treatment group'), the no $A b l$ transgene condition was compared to each $\mathrm{Abl}$ transgene. Pvalue adjustment was applied after pooling all comparisons made for the particular dataset.

For counts of ISNb defects (bypass or stop-short), the severity of the bypass phenotype for each embryonic hemisegment was rank-ordered from least to most severe as follows: wild-type, partial bypass and full bypass. 
Similarly, the severity of the stop-short phenotype was rank-ordered from least to most severe as follows: innervation of muscles 12/13 (wild-type), 6/13 and 6/7. Data were pooled for all datasets that use the same control crosses, and fitted to an ordered logit model with bypasses or stop shorts as the response variable, and $A b l$ transgene and genotype (manipulation of Abl pathway genes) as the two predictor variables. The proportional odds assumption was tested using the Brant test [57]. Post-hoc analysis was carried out by comparisons of the estimated marginal means [56] at a threshold of $p \leq 0.05$ with the Holm method of $p$-value adjustment. A preplanned set of treatment-vs-control comparisons were made for each $\mathrm{Abl}$ transgene where the untreated genotype condition (no genetic elements other than $A b l$ transgene and driver) was used as the controls (p-value adjustment was applied after pooling all comparisons made for the particular dataset). For the crosses of 1407Gal4 to UAS-Abl transgenes, models were separately fitted for overexpression of $A b l$ transgenes alone (including $A b l \Delta 2 E$ and $A b l \Delta P$ ), and for overexpression of $A b l$ transgenes with manipulation of $A b l$ pathway genes (without $A b l \Delta 2 E$ and $A b l \Delta P$ ). Fitting of separate models was necessary to prevent rank-deficiency.

\section{Results}

The $1 \mathrm{Q}$ region of the $\mathrm{Abl} C$-terminal tail recruits the WRC The $\sim 1100$ residue Abl CTD is critical for Abl function in axon guidance and actin dynamics. The $1 \mathrm{Q}$ region, a $\sim 150$ residue region in the CTD situated directly after the kinase domain (residues 656-799 in isoform RF), is the only region absolutely essential for this function [26]. 1Q may play a role in protein-protein interactions, as it has intrinsic disorder that is predicted to become ordered upon protein binding [26] and has a PxxP motif which may bind SH3 domains $[5,26]$. To examine this hypothesis, we carried out a pulldown with recombinant 1Q fused to glutathione S-transferase (GST-1Q) to isolate 1Q binding partners from Drosophila embryo lysate. Following elution, we separated proteins by SDS-PAGE, excised matched gel fragments in GST (control) and GST-1Q lanes, and identified enriched proteins by liquid chromatography-tandem mass spectrometry (LC-MS/ MS). All pulldowns were carried out in triplicate.

Mass spectrometry analysis detected 197 proteins in our pulldowns, with six proteins significantly enriched in the GST-1Q pulldown (Fig. 1a). The full data is found in Table S1 and Mendeley Data at https://data.mendeley.com/ datasets/mw478mgmzs/1 [58]. Strikingly, the proteins Hematopoietic protein/Kette (Hem) and Specifically Racassociated protein 1 (Sra-1) are the top two hits from the pulldown, and were present in approximately equal amounts at $\sim 26$ and $\sim 23$ peptide counts each (Fig. 1a).
Hem and Sra-1 are well-known components of the actinassociated WAVE regulatory complex (WRC), along with subunits HSPC300, Scar/WAVE and Abelson-interacting protein (Abi) [59]. Neither Abi, a known Abl target, nor the other 2 WRC subunits were found in our pulldown. The remaining 4 proteins enriched in our pulldown consisted of ribosomal and ribosome-associated proteins, which are likely of lesser interest.

Interestingly, Hem and Sra-1 can form a heterodimeric subcomplex that can be separated from the rest of the WRC [60]. This pulldown suggests that the Hem/Sra-1 subcomplex may interact directly with $1 \mathrm{Q}$. To establish the in vivo relevance of the putative Abl 1Q interaction with Hem and Sra-1, we elected to first use a genetic approach asking if these genes cooperate in the formation of both motoneuron projections and midline guidance.

\section{Hem and Sra-1 interact genetically with Abl in axon guidance}

Embryonic axon tracts were visualized by immunohistochemistry with mAb 1D4 (anti-Fas2), which labels three longitudinal fascicles in the nerve cord, and motoneurons that project into the periphery. In late-stage embryos (stage 16-17 as previously defined [61]), the longitudinal fascicles do not cross the midline (Fig. 1b). Embryos heterozygous for $\mathrm{Hem}$, Sra-1 or $\mathrm{Abl}$ are wildtype, as are double heterozygote combinations. Embryos homozygous for $\mathrm{Hem}$ or $\mathrm{Abl}$ have occasional axon bundles that inappropriately cross the midline (Fig. 1c, d). The midline defects in Hem homozygous embryos, however, appear qualitatively different from $\mathrm{Abl}$ midline defects, compounding our ability to quantify these differences. Basically, Hem crossing overs are intermingled with narrowing of the nerve cord and thinning of fascicles, while $A b l$ crossing overs appear as distinct bundles of axons. Strikingly, homozygous loss of both $\mathrm{Hem}$ and $\mathrm{Abl}$ severely enhances midline defects, as fascicles become highly disrupted, and axons at the commissures often fail to cross or cross inappropriately (Fig. 1f). Interestingly, in the homozygous/heterozygous combinations, heterozygosity of $\mathrm{Abl}$ rescues Hem loss, although heterozygosity of $\mathrm{Hem}$ does not in turn rescue $\mathrm{Abl}$ loss (Fig. 1e). Below, in the context of evaluating our 1Q mutants, midline crossing overs are counted for heterozygosity of $\mathrm{Hem}$ or $\mathrm{Sra}-1$ in $\mathrm{Abl}$ homozygotes in Fig. 4 and Tables S4-5.

These results are mirrored in the motoneurons at the periphery, where embryos homozygous for $\mathrm{Hem}$ or $\mathrm{Abl}$ have occasional defects in motoneurons (Fig. 1h-l, also see Fig. 3) However, motoneuron projections in $\mathrm{Hem}$ Abl double homozygotes are grossly abnormal with axons frequently misrouted or failing to extend (Fig. 11). Clearly, Hem and $\mathrm{Abl}$ both play important roles in CNS 


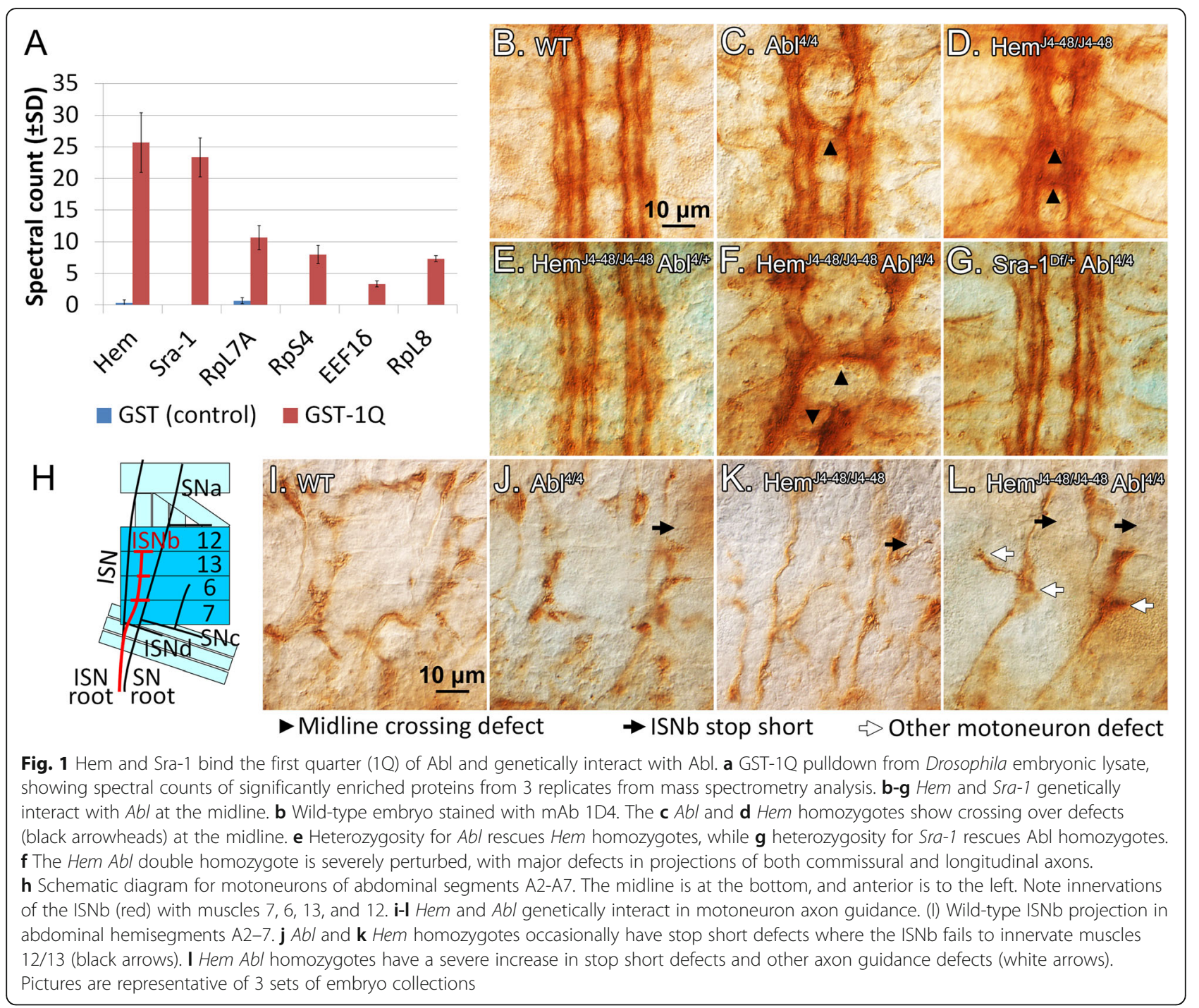

and motoneuron axon guidance, although the severity of the double homozygous phenotype makes it difficult to draw more specific conclusions.

For Sra-1, we used a deletion that removes the Sra-1 gene as well as several neighboring genes $\left(D f(3 R)\right.$ Exel6174, hereby Sra-1 $\left.{ }^{D f}\right)$. Given this limitation, we only used this mutation in its heterozygous state. Importantly, heterozygosity for Sra-1 has no effect on development, but significantly suppresses the midline crossing defects observed in $\mathrm{Abl}$ mutant embryos (Fig. $1 \mathrm{~g})$. These data confirm the importance of $\mathrm{Abl}$, Hem and Sra-1 in cooperating with each other during formation of these axon tracts. Although genetic suppression may occur whether Hem/Sra-1 and Abl function in parallel or within the same pathway, the physical interaction observed in our pulldown points towards the latter explanation. If so, we predict that this genetic interaction would depend on the $1 \mathrm{Q}$ region, and sought to test this idea using a known gain-of-function phenotype of $\mathrm{Abl}$.
Hem and Sra-1 cooperate with Abl in motoneuron axon guidance

In the abdominal segments A2 to A7 [62], motoneuron axon guidance in the intersegmental nerve $\mathrm{b}$ (ISNb) is sensitive to both loss and gain in Abl levels [63]. Overexpression of wild-type $A b l$ in all neurons using the

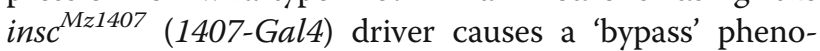
type in the ISNb in about half of hemisegments, where the ISNb fails to defasciculate from the intersegmental nerve (full bypass) or separates at a further point (partial bypass, Fig. 2b-d) [63]. The ability of wild-type Abl to induce these guidance defects allowed us to first ask which of a battery of $A b l$ transgenes deleting all or part of $1 \mathrm{Q}$ recapitulates the bypass phenotype. Briefly, the deletions are as follows: entire first quarter $(\Delta 1 \mathrm{Q})$, first eighth $(\Delta 1 \mathrm{E})$, second eighth $(\Delta 2 \mathrm{E})$ and PxxP motif $(\Delta \mathrm{P}$; see Fig. 2a for delineation of regions). These transgenes are inserted into the same Phi-C31 integrase site ( $\mathrm{ZH}-22 \mathrm{~A})$ and are expressed at a similar level (Fig. S1 A) [26]. 


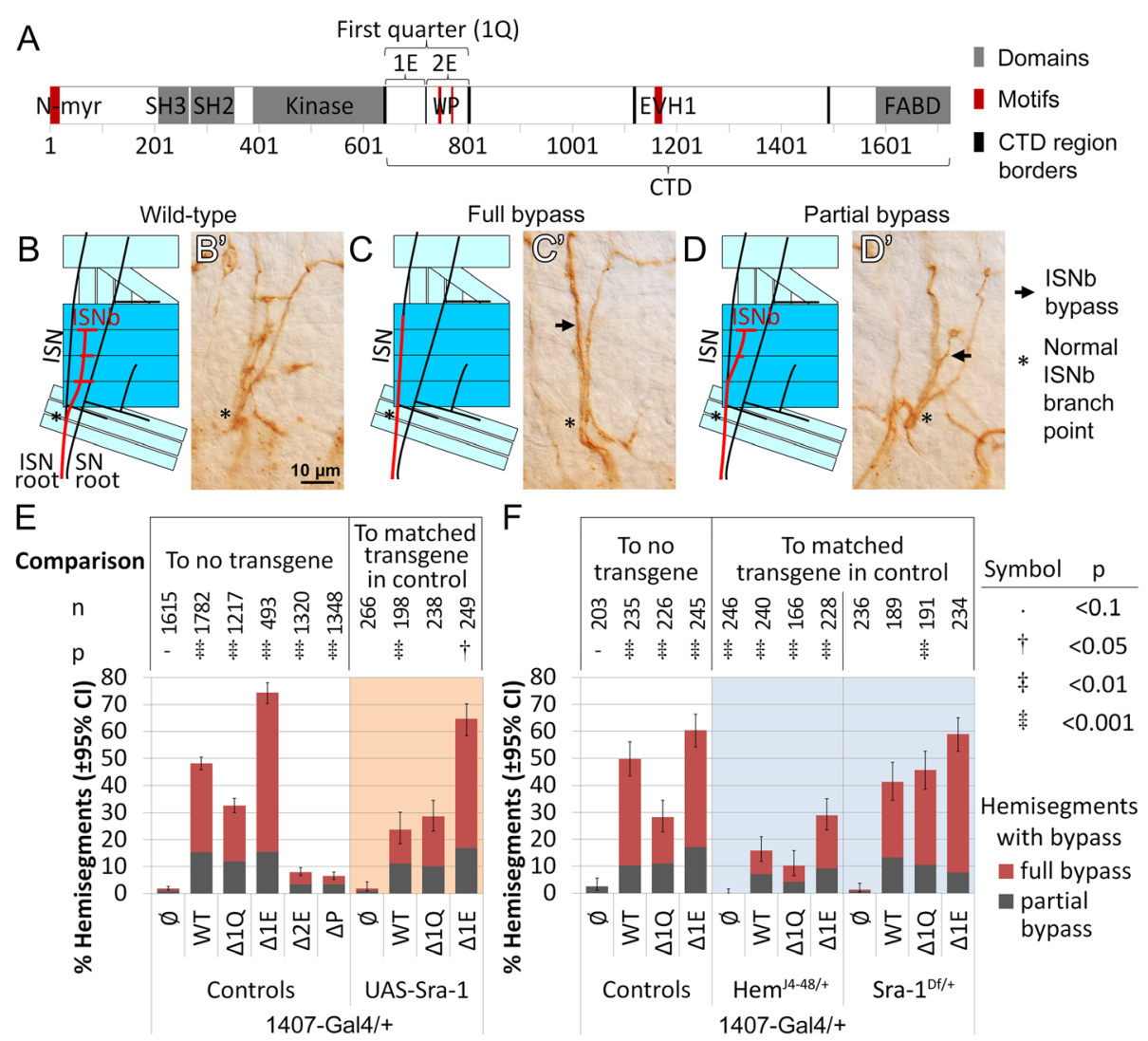

Fig. $2 \mathrm{Hem}$ and Sra-1 modify an Abl gain-of-function ISNb bypass phenotype. a Diagram of Abl, showing domains in grey, and WIRS (W), PXxP (P) and EVH1-binding (EVH1) motifs in red. b \& $\mathbf{b}^{\prime}$ The ISNb (red) defasciculates from the intersegmental nerve (ISN) at a choice point $\left(^{*}\right)$.

Overexpression of Abl causes ISNb bypass defects. c \& $\mathbf{c}^{\prime}$ In a full bypass, the ISNb (arrow) fails to defasciculate from the ISN, while $\mathbf{d} \& \mathbf{d}$ ' in a partial bypass, the ISNb (arrow) defasciculates at a point more distal than expected. e ISNb bypass counts for Abl transgenes (WT, $\Delta 1 \mathrm{Q}, \Delta 1 \mathrm{E}$ or no transgene, $\varnothing$ ) expressed with 1407-Gal4, and interaction of these transgenes with gain of Sra-1. $\mathbf{f}$ Bypass counts for interaction of Ab/ transgenes expressed with 1407-Gal4 with heterozygous loss of Hem or Sra-1. All counts are of individual hemisegments from 10 or more embryos, repeated over 3 or more sets of embryo collections; the n-value represents total hemisegments counted. Confidence intervals given are for penetrance of the phenotype. The direction of crosses is reversed between $\mathbf{e}$ and $\mathbf{f}$ due to a balancer-induced maternal effect (see methods)

Only wild-type $A b l, A b l \Delta 1 Q$ and $A b l \Delta 1 E$ are capable of causing bypass, at 48,33 and $74 \%$ of hemisegments, respectively, while deletion of $2 \mathrm{E}$ or PxxP greatly reduces bypasses to $<10 \%$. These are intriguing data pointing to a major but complex role for $1 \mathrm{Q}$. That is, bypass appears to require signaling from the $2 \mathrm{E}$ region (including the PxxP motif) but $1 E$ influences its use: removal of only $1 \mathrm{E}$ greatly enhances bypass, while removal of the whole $1 \mathrm{Q}$ region lowers the efficacy of Abl to induce bypass. Might this reflect a role for the 1Q region in interacting with Hem or Sra-1? If so, our 1Q transgenes are predicted to be selectively sensitive to alterations in the dosage of either Hem or Sra-1 (overexpression or heterozygous loss). Importantly, neither homozygous loss nor overexpression of $\mathrm{Hem}$ or $\mathrm{Sra}-1$ are known to cause ISNb bypass on their own [64]. Thus, the ability of gain or loss of these proteins to alter ISNb bypass levels would likely indicate a specific role with $\mathrm{Abl}$, perhaps through $1 \mathrm{Q}$.
In this assay, we genetically perturbed $\mathrm{Hem}$ and $\mathrm{Sra}-1$ while overexpressing wild-type $A b l, A b l \Delta 1 Q$ and $A b l \Delta 1 E$, as only these proteins cause significant bypass. In these crosses, the female flies initially carried all lossof-function mutations. However, as we observed unexpected axon guidance defects, likely from a TM6 balancer-induced maternal effect [48], we used the reverse direction with heterozygous loss of $\mathrm{Sra}-1$ or $\mathrm{Hem}$ (and later trio) as explained in methods.

A heterozygous loss of Hem significantly suppresses the bypass phenotype for all 3 transgenes: from 50 to $16 \%$ for wild-type $A b l$, from 28 to $10 \%$ for $A b l \Delta 1 Q$, and from 60 to 29\% for Abl $\triangle 1 E$ (Fig. 2f). Thus, while the levels of $\mathrm{Hem}$ are important for Abl to generate a bypass, we could not detect a specific requirement for the $1 \mathrm{Q}$ region, The function of Hem in the WRC may be structural [59], suggesting that a partial loss of Hem leads to overall destabilization of the complex, and perhaps a general reduction in the WRC's actin polymerization activity. 
On the other hand, the effect of both gain and loss of Sra-1 is selectively sensitive to alterations in the $1 \mathrm{Q}$ region. Overexpression of Sra-1 reduces the ability of wild-type $A b l$ to cause bypass (from 48 to $24 \%$ ) as well as $A b l \Delta 1 E$, albeit less well (from 74 to 64\%). However, Abl $\Delta 1 Q$ continues to cause bypass at similar levels (from 33 to $28 \%$, Fig. 2e). In support, removing half of Sra-1 does not change bypass for wild-type $\mathrm{Abl}$ (from 50 to 41\%) and Abl $1 E$ (from 60 to 58\%), but enhances bypass with expression of $A b l \Delta 1 Q$ (from 28 to 46\%, Fig. 2f). These data suggest that the $1 \mathrm{Q}$ region aids in Abl's ability to function with Sra-1, although the genetic interactions do not necessarily support a simple activation/repression mechanism.

Given that Hem and Sra-1 are tightly linked in a heterodimeric subcomplex $[59,60,65]$, yet only Sra- 1 mutations exhibit sensitivity to mutations in $1 Q$, we suspect that Sra- 1 interacts with the $1 \mathrm{Q}$ region and brought Hem with it in our pulldown assay. This remains to be determined at a biochemical level, but interestingly, the $2 \mathrm{E}$ region includes a PxxP motif which may be recognized by the Abi subunit of the WRC [36, 37], and a putative WRC interacting receptor sequence (WIRS) motif [66] which may bind to a Sra-1/Abi dimer (Fig. 2a). If Abl binds to the Sra-1/Abi interface, it would be in an ideal location to modulate WRC activity, as Sra-1 plays a key role in regulating the WRC's actin polymerization activity through Scar [59], and may be modulated or activated by two Abl partners, Abi and Trio (through Rac) $[66,67]$.

Although we did not detect Abi or Trio in our pulldown, it seemed likely that Abi and perhaps Trio may participate in Abl's regulation of the WRC, a hypothesis we set out to test in loss of function $A b l$ mutants. Zygotic loss of $A b l$ results in a significant reduction in the levels of endogenous Abl, leading to well-documented defects in motoneuron projections and midline guidance. If $\mathrm{Abl}$ interacts with Abi and/or Trio to regulate WRC (via Sra-1), altering the dose of these target genes are expected to alter axon guidance and interact with our $A b l$ transgenes in a 1Q-dependent fashion.

\section{$1 \mathrm{Q}$ is required for $\mathrm{Abl}$ regulation of WRC during ISNb extension}

Homozygous loss of $\mathrm{Abl}$ causes defects in axon guidance in the CNS and motoneurons, including 'stop short' of the ISNb [16]. In abdominal segments A2-A7 of latestage embryos, the ISNb innervates 3 clefts between the muscles 6, 7, 12 and 13 (Fig. 3a, b). In the stop short phenotype, the ISNb fails to reach the muscle $12 / 13 \mathrm{cleft}$ (6/13 stop short), or more rarely, the muscle $6 / 13 \mathrm{cleft}$ (7/6 stop short) [16]. Loss of WRC components including Sra-1 [68], Abi and Scar [21] are known to cause stop short defects, suggesting that branched actin polymerization from WRC activity is necessary for ISNb extension. If so, might the $\mathrm{Abl} 1 \mathrm{Q}$ region modulate Sra1 and its partners in this context?

In our hands, the $A b l^{4 / 2}$ null condition causes 'stop short' defects in $\sim 20 \%$ of hemisegments (Fig. 3g), close to previous reports using this allelic combination [16]. When expressed with 1407-Gal4, stop shorts are rescued by a wild-type $A b l$ transgene (from 19 to $4 \%$ ) as expected (Fig. $3 g$ ). In contrast, rescue is incomplete with deletion of $1 \mathrm{Q}, 2 \mathrm{E}$ or $\mathrm{P}(\sim 10 \%$ for all); surprisingly, deletion of $1 \mathrm{E}$ rescues to a similar level as the wild-type transgene (to 5\%). These data demonstrate that $1 \mathrm{Q}$, and especially $2 \mathrm{E}$ and its PxxP, are important for our transgenes to rescue motoneuron projections. As such, we predicted that rescue of stop shorts would also be selectively sensitive to alterations in the dose of the WRC pathway genes Hem, Sra-1, Abi and trio.

Unfortunately, gain of Sra-1, as well as heterozygous loss of trio, Hem or Abi all have little effect on the ability of our $A b l$ transgenes to rescue stop shorts (the majority fall within the $2.5-10 \%$ range), which may reflect in part the limited dynamic range of this assay (Table S2 \& S3). In addition, due to poor stock health, the effect of a heterozygous loss of Sra-1 could only be evaluated using wild-type $A b l$ and $A b l \Delta 1 Q$ transgenes. Heterozygous loss of $S r a-1$ rescues stop shorts in $A b l^{4 / 2}$ embryos (from 18 to $3 \%)$ and does not alter wild-type $A b l$ 's ability to rescue; less but statistically significant rescue is observed when $A b l \Delta 1 Q$ is present (from 20 to $11 \%$ ). These results suggest that excess Sra-1 activity contributes to stop shorts in $\mathrm{Abl}$ mutants, and, consistent with our bypass results, without the $1 Q$ region, Abl has difficulty regulating Sra- 1 .

We also observe a clear requirement for $1 \mathrm{Q}$ for $\mathrm{Abl}$ to regulate over-expressed $A b i$ and trio. Overexpression of $A b i$ does not alter stop short levels in the $A b l^{4 / 2}$ embryos alone, nor does it alter the rescue seen with a wild-type $A b l$ transgene or $A b l \Delta 1 E$ (Fig. 3g). Yet, expression of $A b i$ with $A b l \Delta 1 Q$ nullifies the partial rescue seen with $A b l \Delta 1 Q$ alone (from 9 to $19 \%$ ), suggesting that $A b l \Delta 1 Q$ is deficient in regulating Abi. In comparison, overexpression of trio alone greatly exacerbates stop shorts in $A b l^{4 / 2}$ embryos (from 19 to 63\%; Fig. 3g), but there is a robust suppression of these with expression of wild-type $A b l$ or $A b l \Delta 1 E$ (to $<15 \%$ ). In contrast, $A b l \Delta 1 Q$ fails to rescue the increased stop shorts caused by increased Trio, while $A b l \Delta 2 E$ and $A b l \Delta P$ are partially impaired ( $\sim 35 \%)$. Together these data indicate that 1Q (and especially $2 \mathrm{E}$ and its $\mathrm{PxxP}$ ) are important for Abl to suppress the effects of excess Trio. Note that our observation that overexpression of trio exacerbates stop shorts in $A b l$ mutants contrasts with an earlier report indicating rescue of stop shorts [21]; however, in our hands, the UAS-trio transgene used in the prior work also exacerbates $A b l$ stop shorts (Table S3). 

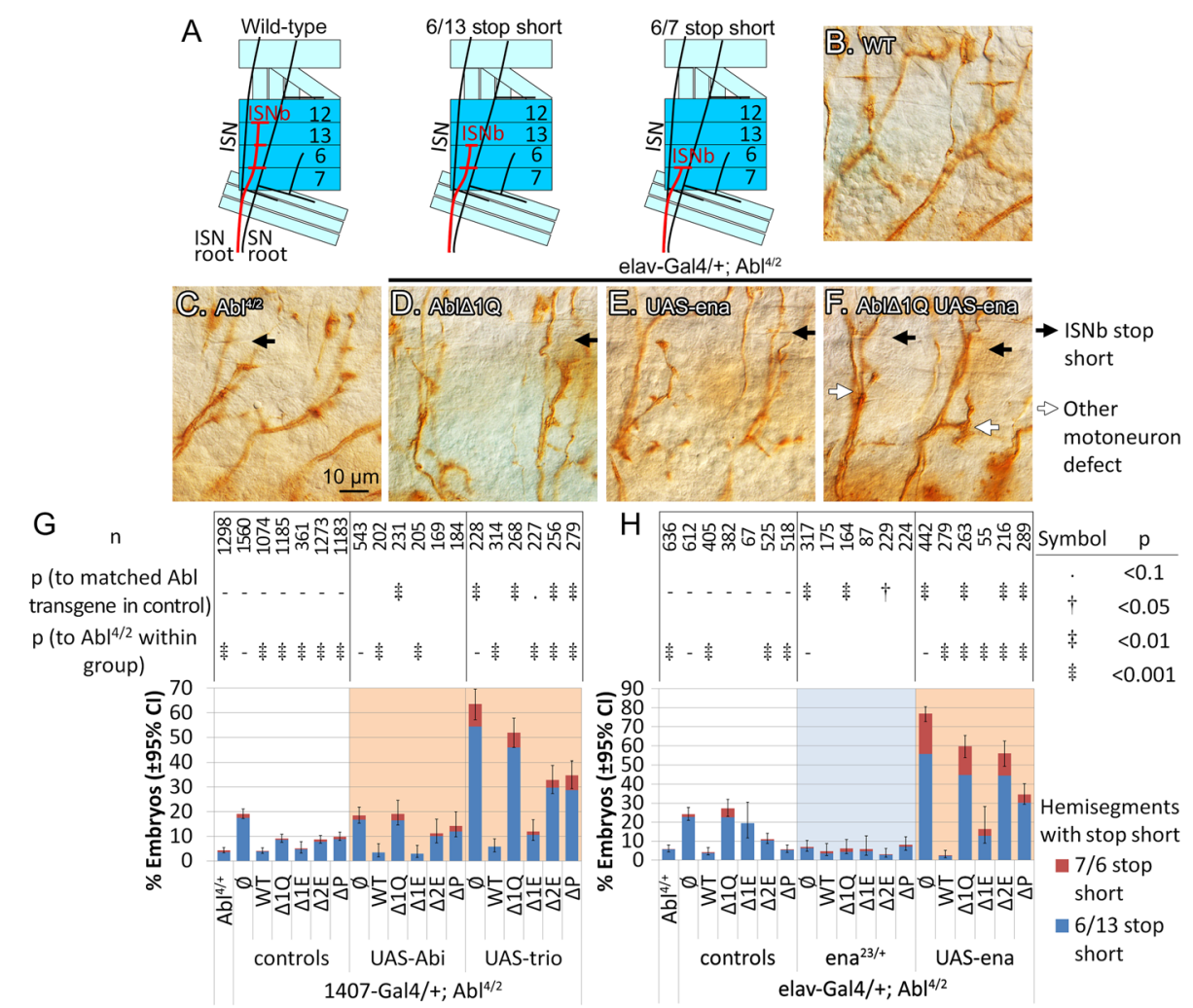

Fig. 3 The $1 \mathrm{Q}$ region of Abl is required for Abi and Trio genes to modify ISNb stop shorts in Ab/ loss-of-function mutant embryos. a The ISNb innervates the clefts between muscles $6 / 7,6 / 13$ and 12/13. In Ab/ mutants, the ISNb occasionally fails to innervate the 12/13 cleft (6/13 stop short) or 6/12 cleft (6/7 stop short). b Wild-type ISNb innervations. d Ab/ mutant showing ISNb stop shorts (black arrow indicates 12/13 cleft). Expressing either $\mathbf{d} A b / \Delta I Q$ or e ena increases stop shorts in $A b /$ mutants. $\mathbf{f}$ Expression of both does not further increase stop shorts but causes variable misrouting or fasciculation defects in motoneuron projections (white arrows). $\mathbf{g}$ Stop short counts in Ab/ mutants expressing Abl transgenes, with overexpression of Abi or trio with 1407-Gal4. h Stop short counts with heterozygous loss and overexpression of ena with elavGal4. All counts are of individual hemisegments from 10 or more embryos, repeated over 3 or more sets of embryo collections; the n-value represents total hemisegments counted. Confidence intervals given are for penetrance of the phenotype

Putting these results together, the $1 \mathrm{Q}$ region and especially 2E and PxxP, is required for Abl to suppress, or at least tightly control, the activity of Sra-1, Abi and Trio. A failure to do so results in stop short defects, probably reflecting a change in the regulation of branched actin formation [69]. It is intriguing that the $1 \mathrm{Q}$ region was sufficient to pull down Sra-1 (and Hem), and aids in the regulation of Sra-1, Abi and Trio during motoneuron extension. Future biochemistry will need to evaluate the full nature of a potential complex, including the individual roles of Abl, Sra-1, and Abi (and indirectly, Trio). As described below, our analysis with Enabled (Ena) suggests that it, too, may need to be included.

\section{Abl regulates Ena through $1 Q$}

While the WRC is thought to control branched actin formation, Ena may work in a parallel pathway to regulate linear actin polymerization [21]. Together, these pathways regulate growth cone extension in response to guidance cues. Ena is well established as a binding partner and phosphorylation target of Abl [19, 22]. Genetically, heterozygosity of ena suppresses the axon guidance defects from homozygous loss of $A b l[18,19]$. There is evidence that this genetic suppression stems from ectopic localization of Ena when $\mathrm{Abl}$ is lost [70, 71], which can then be alleviated by heterozygosity of ena. Given that Ena is recruited to the SH3 domain of $\mathrm{Abl}$ [22], we predict that an interaction between $\mathrm{Abl}$ and Ena would not involve 1Q, leaving Ena properly localized. Put another way, our 1Q mutant transgenes should be insensitive to a gain or loss of ena in these embryos. Unexpectedly, Abl $1 \mathrm{Q}$ mutant transgenes also appear to be defective in functioning with Ena.

To test for Ena's role, we needed to use the pan-neural elav-Gal4 driver instead of 1407-Gal4, as the 1407-Gal4 insertion is in close proximity to ena. The elav-Gal4 driver expresses at roughly 2-fold higher than 1407-Gal4 (Fig. S1 B), and this slightly alters the rescue of stop shorts by $A b l$ transgenes: from a baseline of $24 \%$ stop shorts in $A b l^{4 / 2}, A b l \Delta 1 Q$ and $A b l \Delta 1 E$ now fully fails to 
rescue $\mathrm{Abl}$ loss, at 27 and 19\% respectively (Fig. $3 \mathrm{~h}$ ). This difference compared to 1407-Gal4 suggests that loss of $1 \mathrm{E}$ renders the Abl transgene more sensitive to expression levels, perhaps pointing to a role of $1 \mathrm{E}$ in limiting or fine-tuning Abl function; we also observe this at the midline (see further below). Rescue with other transgenes remain similar to that observed using the 1407-Gal4 driver.

Consistent with previous genetic results at the midline $[18,19]$, removing one copy of ena suppresses ISNb stop shorts in $A b l^{4 / 2}$ embryos (from 24 to 7\%). Likewise, removing half of ena improves the level of stop shorts seen with $A b l \Delta 1 Q$ and $A b l \Delta 2 E$ (from 27 to $6 \%$ and from 11 to $3 \%)$, indicating that excess Ena activity still contributes to stop shorts when $\mathrm{Abl} \Delta 1 \mathrm{Q}$ and $\mathrm{Abl} \Delta 2 \mathrm{E}$ are present. In contrast, loss of ena does not alter the rescue observed by wild-type $A b l, A b l \Delta 1 E$ and $A b l \Delta P$ (5-8\% for all). Thus, at least $\mathrm{Abl} \Delta 1 \mathrm{Q}$ and $\mathrm{Abl} \Delta 2 \mathrm{E}$ have trouble regulating Ena.

Overexpression of ena confirms the importance of $1 \mathrm{Q}$ in regulating Ena (Fig. 3b-f, h). Like trio above, overexpression of ena greatly exacerbates stop shorts in $A b l^{4 / 2}$ null embryos (from 24 to $77 \%$ ), and this is essentially rescued by a wild-type $A b l$ transgene (to $3 \%$ ). Interestingly, $A b l \Delta 1 E$ readily suppresses stop shorts induced by overexpressed Ena (from 77 to $15 \%$ ) while $A b l \Delta 2 E$ and $A b l \Delta P$ show a stepwise ability to rescue (from 77 to $56 \%$ and 35\% respectively). This difference supports an important role for $2 \mathrm{E}$ and PxxP in regulating Ena. It is worth noting that the complete removal of both $1 \mathrm{E}$ and $2 \mathrm{E}$, in our Abld1Q mutant, allows a slight decrease in stop shorts (from 77 to 60\%), but also induces variable misrouting or fasciculation defects in other motoneuron branches (Fig. 3f). This data suggest that the $1 \mathrm{E}$ region plays a (undefined) role in determining how Abl interacts with Ena.

Both the gain and loss of ena data suggest that Abl requires the $1 \mathrm{Q}$ region (especially $2 \mathrm{E}$ and PxxP) to suppress Ena activity. This was unexpected as $\mathrm{Abl}$ is thought to bind to Ena via the $\mathrm{SH} 3$ domain, and possibly the EVH1-binding motifs in another part of its Cterminal domain. As Ena and Sra-1 are both regulated by the same $1 \mathrm{Q}$ region of $\mathrm{Abl}$, we speculate that during these motoneuron guidance events, Abl regulates Ena via its role with the WRC (see discussion section). Given this surprising result, we elected to explore the role of 1Q during midline guidance, where the genetic interaction of ena and $A b l$ has largely been examined $[8,10$, 19]. The midline also allows us to test the role of $1 Q$ in a different signaling context, as receptors upstream of $\mathrm{Abl}$ at the midline are likely not the same as those used in the ISNb $[7,8,12,13]$.

\section{Axon guidance at the midline also requires $1 \mathrm{Q}$}

Late-stage embryos stained with mAb 1D4 display three longitudinal fascicles in the nerve cord. In $A b l^{4 / 2}$ homozygotes, roughly half of embryos show midline crossing over defects, where these fascicles will inappropriately cross the midline. As with ISNb stop shorts, we used the frequency of this phenotype to assess the function of our $A b l$ mutant transgenes, and the role of $1 \mathrm{Q}$ with WRC proteins and Ena. As above, we predict that alterations in the 1Q region will be sensitive to gain or loss of these genes. Note that the penetrance of midline crossing overs is presented here as a proxy for the severity of the phenotype, but embryos may also display varying number of crossing overs (shown by color and accounted for in statistics); these will be noted when appropriate. The tabulated data is found in Tables S4-6.

In $A b l^{4 / 2}$ mutant embryos, roughly $50 \%$ of embryos show midline crossing overs, at roughly 1-2 crossing overs per embryo. Abl function at the midline remains highly dependent on $1 \mathrm{Q}$, especially $2 \mathrm{E}$ and its PxxP motif. With 1407-Gal4, a wild-type $A b l$ transgene rescues crossing overs to $8 \%$, while $A b l \Delta 1 Q$ fails to provide any rescue (54\%; Fig. 4a). Abl $\Delta 2 E$ and $A b l \Delta P$ only rescue about half as well (24 and $25 \%$ respectively), while $A b l \Delta 1 E$ performs slightly better (16\%). The need for 1Q and especially $2 \mathrm{E}$ regions to rescue crossovers suggests that Abl's ability to function with the WRC is important in this guidance context. If so, the loss of $1 \mathrm{Q}$, and especially $2 \mathrm{E}$ and its PxxP motif are predicted to be sensitive to changing the dosage of the WRC pathway genes Hem, Sra-1, Abi and trio. Our results align with this prediction, but we also observe a larger role of $1 \mathrm{E}$.

With Sra-1, heterozygous loss of Sra-1 greatly rescues the midline crossing overs of loss of $\mathrm{Abl}$ (from 64 to 8\%, Fig. $4 \mathrm{~b})$, suggesting that excess Sra- 1 activity contributes to these defects. Expression of $A b l \Delta 1 Q$ completely prevents this rescue (to $64 \%$ ) but other regions of $1 \mathrm{Q}$ could not be tested due to poor stock health. The converse effect is observed for gain of Sra-1, which slightly suppresses crossing overs with $A b l \Delta 1 Q$ (from 50 to 40\%), although genetic interactions with deletions of $1 \mathrm{Q}$ subregions were not significant (Table S4; see also for $\mathrm{Hem}$ data). Together, these data suggest that excess Sra-1 activity contributes to crossing overs, although the role of the regions of $1 \mathrm{Q}$ remains unresolved. On the other hand, both halves of $1 \mathrm{Q}$ appear to be important in regulating Abi and Trio at the midline.

Heterozygosity of $A b i$ is reported to partially rescue crossing overs in $\mathrm{Abl}$ mutants [20]. Here, removing half of Abi levels in $A b l^{4 / 2}$ embryos trends towards suppression (from 50 to $28 \%$, Fig. 4a, S2), but this did not reach statistical significance. As expected, heterozygous loss of $A b i$ has no effect on rescue by wild type Abl, while helping $A b l \Delta 2 E$ (from 24 to $8 \%$ ) and maybe $A b l \Delta P$ (from 25 to $14 \%)$. On the other hand, loss of Abi now completely prevents $A b l \Delta 1 E$ from rescuing crossing overs (from 16 to 57\%), and even enhances midline crossing overs for 


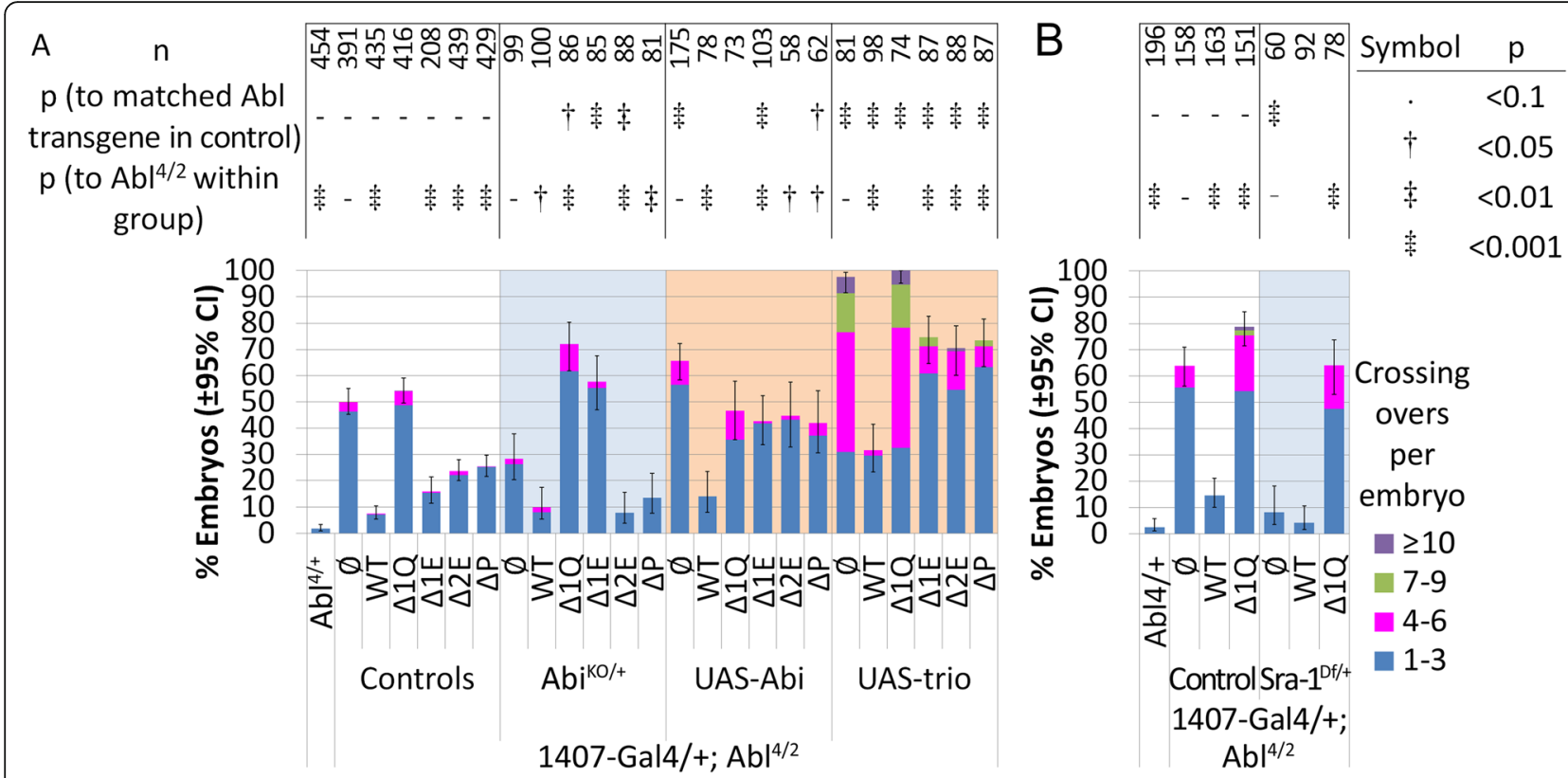

Fig. $4 \mathrm{Abl} 1 \mathrm{Q}$ regulates the WRC at the midline. a Midline crossing overs in Ab/ mutants expressing Ab/ transgenes with 1407-Gal4, with gain or loss of Abi, or gain of trio. b Midline crossing overs in Abl mutants expressing Abl transgenes with 1407-Gal4, with heterozygous loss of Sra-1. Confidence intervals given are for penetrance of the phenotype. All counts are from 3 or more sets of embryo collections, with 30 embryos per set; the n-value represents embryo count. Confidence intervals given are for penetrance of the phenotype. The direction of crosses is reversed between $\mathbf{a}$ and $\mathbf{b}$ due to a balancer-induced maternal effect

Abld1Q (from 55 to $72 \%$ ). Interpreted directly, loss of $2 \mathrm{E}$ or PxxP seems to impair Abl's ability to suppress Abi, but loss of $1 \mathrm{E}$ (in $\triangle 1 E$ and $\triangle 1 Q$ ) instead reverses this effect. A clear molecular explanation for this result is not yet possible, but it does suggest an interplay between $1 \mathrm{E}$ and $2 \mathrm{E}$ regions in governing how this region works (see discussion). Conversely, overexpression of $A b i$ alone in $A b l^{4 / 2}$ embryos increases crossing overs from 50 to $66 \%$, but expressing wild type $A b l$ with $A b i$ still rescues most crossovers (14\%). On the other hand, when expressed with $A b i$, all $1 \mathrm{Q}$ mutant transgenes have difficulty bringing the over-expressed Abi under control-for $\mathrm{Abl} \triangle 1 E, \triangle 2 E$ and $\triangle P$, overexpression of Abi increases embryos with crossing overs to $42-45 \%$, while level of crossing overs remain high and unchanged for $\mathrm{Abl} \Delta 1 \mathrm{Q}$. These results suggest that Abl inhibits Abi activity in order to prevent crossovers, and while $2 \mathrm{E}$ and $\operatorname{PxxP}$ are involved, the $1 \mathrm{E}$ region also exerts a regulatory effect.

Likewise, overexpressing trio in $A b l^{4 / 2}$ mutants results in a large increase in the frequency and expressivity of midline crossovers, but these are brought under control by co-expressing wild type $A b l$. As observed with Abi, all of our 1Q mutant transgenes have difficulty bringing excess Trio under control, resulting in significant numbers of crossovers (Fig. 4a).

Clearly, the $1 \mathrm{Q}$ region is important for the function of Abl with the WRC during midline guidance events. Similar to that observed with motoneurons, 2E and PxxP remain the most important regions of $1 \mathrm{Q}$ to regulate both Abi and Trio during midline guidance. But at the midline, the $1 \mathrm{E}$ region seems to play a larger role, seemingly to influence how $2 \mathrm{E}$ communicates with these targets. This may reflect the specific axon guidance pathways utilizing $\mathrm{Abl}$ at the midline, or a greater need for preciselyregulated $\mathrm{Abl}$ activity for axons to navigate the midline.

\section{Ena regulation at the midline requires $1 Q$}

Having established the requirement of $1 \mathrm{Q}$ and the WRC at the midline, we next turned to Ena. Heterozygous loss of ena suppresses midline crossing defects of $A b l$ mutant embryos $[8,10,19]$, suggesting that Abl inhibits Ena to facilitate midline crossing. Based on our motoneuron data, and contrary to our original expectations, the $1 \mathrm{Q}$ region, including $2 \mathrm{E}$ and its $\mathrm{PxP}$ should be important for Abl to regulate Ena at the midline.

Recall that for Ena, we had to use the elav-Gal4 driver instead of 1407-Gal4. Expression of $\mathrm{Abl}$ transgenes in $A b l^{4 / 2}$ embryos with elav-Gal4 ( 2-fold stronger than 1407-Gal4) reveals an expression level effect that highlights the importance of $1 \mathrm{E}$. Counts for most $\mathrm{Abl}$ transgenes remain similar to 1407-Gal4 (Fig. 5i \& Table S6), but here both $A b l \Delta 1 Q$ and $A b l \Delta 1 E$ now greatly exacerbate crossing overs to 95 and $83 \%$ respectively (Fig. $5 \mathrm{~d}$, i). This

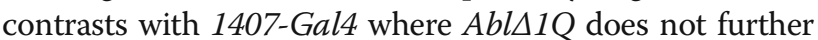
perturb $A b l^{4 / 2}$ crossing overs, while $A b l \Delta 1 E$ rescues them. We speculate that the increased sensitivity to the dose of 
transgene reflects the different signaling pathways converging on $1 \mathrm{E}$ to fully regulate $\mathrm{Abl}$ activity at the midline. Nevertheless, we could still evaluate whether our Abl transgenes selectively regulate Ena at the midline.

In $\mathrm{Abl}$ null embryos, heterozygous loss of ena rescues crossing overs from 43 to $16 \%$ (Fig. 5e, i), suggesting that excess Ena contributes to midline defects, as observed by others [19]. Removal of half of ena rescues crossing overs when $A b l \Delta 2 E$ is expressed (from 48 to $18 \%$ ), while $A b l \Delta P$ remains relatively strong in its ability to rescue (20\%). On the other hand, removing half of Ena has only a small effect on the large number of crossing overs observed with $A b l \Delta 1 Q$ or $A b l \Delta 1 E$ expression, suggesting that these crossing overs may reflect more than a loss in Ena regulation. These results point to a role for $2 \mathrm{E}$ in suppressing Ena at the midline, a notion more clearly demonstrated when we overexpress Ena in Abl mutants.

Overexpression of ena in the $A b l^{4 / 2}$ null greatly increases midline crossing overs, from 43 to $99 \%$, although this is essentially reverted when a wild-type $A b l$ transgene is re-expressed (Fig. 5g, i). Expression of Abl $\Delta 1 Q$ completely fails to revert ena overexpression; penetrance remains high at $100 \%$ and crossing overs per embryo are further elevated (Fig. 5h, i). In comparison, $A b l \Delta 2 E$ and $A b l \Delta P$ perform slightly better but are still clearly impaired: while the penetrance of crossing overs remains high at 100 and $78 \%$, the number of crossing overs per embryo decreases compared to ena overexpression alone (Fig. 5i). Gain of ena clearly demonstrates a requirement for $1 \mathrm{Q}$, and especially $2 \mathrm{E}$ and PxxP, in order for Abl to suppress Ena during midline guidance.

Surprisingly, Abl $\Delta 1 E$ rescues about half of midline crossing overs (to $48 \%$ ), and is clearly better than $A b l \Delta 2 E$ and $A b l \Delta P$ in bringing Ena under control. This contrasts with observations with $\mathrm{Hem}, \mathrm{Abi}$ and trio where all 3 of these $A b l$ transgenes perform at about the same level. That is, at the midline, removal of $1 \mathrm{E}$ has little impact on Abl's ability to regulate Ena, yet $1 \mathrm{E}$ is required to regulate the WRC genes, which also require $2 \mathrm{E}$ and $\mathrm{P}$. Thus, as detailed in the discussion, we hypothesize that $1 \mathrm{E}$ plays a role in biasing how $\mathrm{Abl}$ works with Ena or the WRC.

\section{Discussion}

Our pulldown and genetic analyses provide some of the first insight into why the $1 \mathrm{Q}$ region of $\mathrm{Abl}$ is so important for its function. 1Q physically interacts with Hem and Sra-1, likely forming a link between Abl and the WRC. Our detailed genetic analyses with multiple different axon guidance endpoints confirm the importance of 1Q in Abl's ability to function with these proteins and their partners in axon guidance, and further point towards the $2 \mathrm{E}$ and its PxxP motif as the most critical regions for this role.
Overall, the regions of $1 \mathrm{Q}$ contribute the following functions to Abl's role in axon guidance: The $2 \mathrm{E}$ region and its PxxP motif are a core requirement for Abl, with deletion of either region having similar deleterious impact (removal of PxxP tends to be slightly milder). $A b l$ transgenes with either $2 \mathrm{E}$ or PxxP removed are impaired in their ability to rescue axon guidance phenotypes in $A b l$ mutant embryos, and further lose the ability to cause the gain-of-function ISNb bypass phenotype. As we discuss further below, the $2 \mathrm{E}$ region and its PxxP motif are also prime candidates to mediate proteinprotein interactions with the WRC. Removal of 1E, on the other hand, results in an $A b l$ transgene that still partially or nearly fully rescues $A b l$ loss, but rescue is now more sensitive to the level of $A b l$ transgene expression (e.g. compare 1407-Gal4 vs elav-Gal4 at the midline) and the guidance event examined. For example, in the gain-of-function bypass phenotype, overexpression of $A b l \Delta 1 E$ suggests that this protein is 'overactive', as it causes more defects than a wild-type transgene. Yet at the midline, $\mathrm{Abl} \Delta 1 \mathrm{E}$ does not seem to be hyperactive, as it clearly suppresses ena overexpression at the midline and is poorer at suppressing trio or Abi overexpression. Taken together, we hypothesize that $1 \mathrm{E}$ may control the target selectivity of Abl, biasing an interaction towards Ena over WRC, perhaps in the context of the specific guidance choice point being investigated.

The hallmark of the 2E region is a PxxP motif, which the vertebrate literature suggests multiple candidates for binding, notably Abi, Crk, Nck, and Grb [33-38]. Here, we coupled a GST pulldown from embryo lysate with mass spectrometry for protein identification to provide an unbiased sampling of $1 \mathrm{Q}$ binding partners. We identified Hem and Sra-1 in roughly equal amounts, consistent with their known association as a heterodimeric 'subcomplex' $[59,60]$. Double homozygous embryos of $\mathrm{Abl}$ and $\mathrm{Hem}$ display numerous guidance defects in the nerve cord scaffold and motoneuron projections. This genetic interaction was not previously documented, although perhaps not surprising given the importance of both Abl and Hem in actin dynamics. These proteins may also have an antagonistic relationship. Firstly, heterozygous loss of Sra-1 rescues the midline crossing defects of $\mathrm{Abl}$ mutants, and similarly heterozygous loss of $\mathrm{Abl}$ suppress crossovers observed in Hem mutants. As homozygous loss of either $\mathrm{Hem}$ or $\mathrm{Sra}-1$ alone has been reported to cause midline defects similar to loss of $A b l$ [64], the apparent antagonistic relationship may not reflect a simple on/off switch. Instead, we speculate that Abl selectively controls the WRC either in response to upstream receptors and/or affects the WRC's subcellular localization, as observed with another Abl partner, Ena [71, 72]. Over-expressing $A b l$ in motoneurons further supports the involvement of $\mathrm{Hem}$ and Sra-1 in Abl 


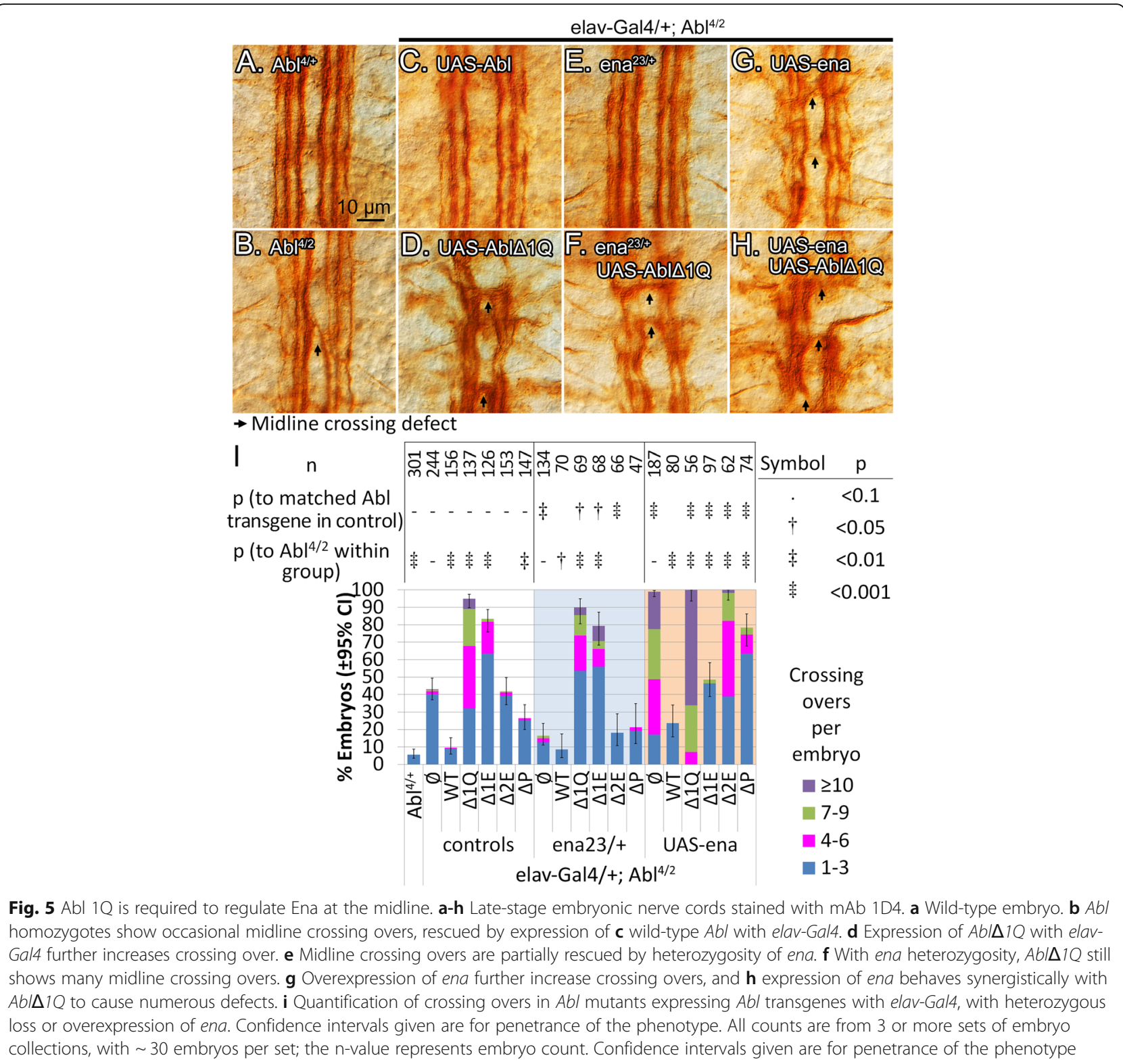

function, and a role for 1Q. Heterozygous loss of Hem unilaterally suppresses the bypass phenotype caused by $A b l$ transgenes, whether or not 1Q is present, perhaps doing so through its partner, Sra-1, or by dictating the overall level of the WRC. In contrast, the effect of lossand gain-of-function Sra-1 mutations on bypass is sensitive to alterations in $1 \mathrm{Q}$, hinting that Sra-1 may play a more direct role with this region.

The role of $1 \mathrm{Q}$, however, is likely not limited to Hem and Sra-1. Along with both proteins, Abi, Scar/WAVE and HSPC300 are also core members of the WRC. However, these 3 remaining members were not identified in our pulldown, which could reflect our pulldown conditions (medium stringency due to nonionic detergent and moderate salt concentrations to facilitate embryo lysis) or the labile nature of regulatory interactions, which may be difficult to detect in pulldowns. Of the remaining 3 WRC members, $\mathrm{Abi}$ is the best candidate for a direct function with $1 \mathrm{Q}$, as $\mathrm{Abi}$ interacts with $\mathrm{Abl}$ during axon guidance, and is thought to play a modulatory role with the WRC $[73,74]$. Moreover, the $2 \mathrm{E}$ region (the CR1 region of Rogers et al. [5]) contains the PxxP motif that may bind Abi, is conserved among invertebrates, and is important for Abl's role in several actin-based processes [5]. We further identify a putative WIRS motif in 2E of Abl (Fig. 2a), which may interact with Sra-1 and Abi [66]. Indeed, 2E and its PxxP are both important for Abl to bring overexpressed Abi under control at the midline and in motoneurons. Interestingly, heterozygous loss of $A b i$ shows unique genetic interactions with $1 \mathrm{Q}$ mutants at the midline-it 
exacerbates defects with $A b l \Delta 1 Q$ and $\Delta 1 E$ while instead partially rescuing defects with $A b l \Delta 2 E$. This result cannot be explained by a simple activation/repression mechanism. We speculate that this instead reflects a role of Abi with $1 \mathrm{E}$ in our hypothesized Abl target selection function, perhaps in relation with Ena (also see further below). Finally, the 1Q region, through Trio, also appears to impact the function of Rac GTPase as an upstream activator of the WRC. Rac is considered a major regulator of the WRC by direct binding of active Rac to Sra-1, allowing Sra-1 to activate the actin polymerization activity of Scar/WAVE [59, 75]. This may be reflected in our results with the Trio GEF (an activator of Rac), which exacerbates midline and motoneuron defects when overexpressed in $\mathrm{Abl}$ mutants. We observe that $\mathrm{Abl}$ requires $2 \mathrm{E}$ and PxxP to bring the effects of overexpressed Trio under control.

Surprisingly, we also find that $1 \mathrm{Q}$ is required for $\mathrm{Abl}$ to regulate Ena, despite no indication that $1 \mathrm{Q}$ can interact directly with Ena. This finding is all the more surprising given that the putative Ena-binding motifs elsewhere in the CTD are dispensable for Abl function [5]. Like Trio and Abi, Abl requires $2 \mathrm{E}$ and its PxxP motif to regulate Ena; overexpressing ena in $A b l^{4 / 2} \mathrm{mu}$ tants causes midline and motoneuron axon guidance defects that are poorly, if at all, rescued by $A b l$ transgenes lacking 1Q, 2E or PxxP. Conversely, heterozygous loss of ena suppresses midline and motoneuron defects in $A b l^{4 / 2}$ mutant embryos expressing Abl lacking 1Q, 2E or $\operatorname{PxxP}$ to varying degrees, suggesting that misregulation or failure to suppress Ena contributes to the generation of these defects. Interestingly, others have demonstrated that Ena and Abi can interact directly [76], and this interaction plays a non-cell autonomous role in axon guidance of photoreceptor neurons. These authors also suggested that $\mathrm{Abl}$ was a candidate in regulating the Abi-Ena interaction. Our results are consistent with this idea, as both $2 \mathrm{E}$ and PxxP are apparently required to regulate Ena and $\mathrm{Abi}$. Thus, while it remains possible that Ena and the WRC function in parallel downstream of Abl, our data suggests that both pathways are tightly linked by $\mathrm{Abl}$ and that this interaction requires a functional 1Q region. Clearly, future work with genetic analyses manipulating all 3 genes, as well as in vitro biochemistry will be needed to verify this potential regulatory triad.

How might our findings fit in with current ideas on motility and axon guidance? Fundamentally, cell migration is driven by the dynamics of actin-rich protrusions at the cell or growth cone periphery that includes balancing the rates of actin polymerization between branched and linear elongation of $\mathrm{F}$-actin filaments [77]. Importantly, the activity of Arp2/3-the main target of the WRC - is thought to be the major driver of branched Factin nucleation in lamellipodia [78], while Ena appears to be a linear actin elongation factor [79]. The relative amounts of branched nucleation and linear actin elongation determines the ratio of $\mathrm{F}$-actin branches per length of filament, which in turn controls the speed and directional persistence of actin protrusions [77]. In this model, high Arp $2 / 3$ activity should favor directional persistence of lamellipodia, while high Ena activity may favor faster, more dynamic lamellipodia [80]. Based on this framework, Abl's ability to regulate both the WRC and Ena places it in a key position to modulate lamellipodial dynamics, and thus, growth cone steering. This is certainly consistent with the multitude of axon guidance pathways that co-opt $\mathrm{Abl}$ as a downstream effector. Moreover, recent work has begun to characterize the contribution of Abl to growth cone morphology and fluctuations in actin dynamics at a biophysical level [81, 82].

Abl's proposed ability to regulate growth cone steering through actin dynamics helps explain the guidance defects we observe in our assays. The Roundabout (Robo) receptor is likely the most well-studied receptor with respect to Abl signaling, and governs growth cone repulsion at the midline in Drosophila. Currently, Robo is thought to recruit Abl by direct binding, and Abl appears to be a negative regulator of Robo, perhaps via phosphorylation [13]. Yet, Robo also recruits Ena directly, and genetic analyses suggest that Robo's activity in midline repulsion occurs partially through Ena [13]. Thus, Ena and Abl appear to work in opposite directions downstream of Robo. Our results agree with this, as we observe that Abl counters Ena activity in midline crossing through 1Q. In addition, Robo also activates Rac signaling through the Rac GEF Son of Sevenless [83], and in C. elegans at least, Robo likely regulates the WRC through Rac during axon guidance [84]. Thus, Abl, Ena and the WRC are implicated downstream of Robo. While Robo signaling likely involves direct regulation of each protein, the final outcome is probably fine-tuned by 3 -way inter-regulation between $\mathrm{Abl}, \mathrm{WRC}$ and Ena, a process that we predict will require the $1 \mathrm{Q}$ region of Abl. Recent work with vertebrate Robo also suggests a temporal component in Robo signaling - upon initial stimulation by the Slit ligand, Robo induces a transient outgrowth of filopodia through Ena, following which growth cone collapse occurs [85]. It is tempting to speculate that $\mathrm{Abl}$ is coordinating aspects of this regulation through its ability to balance the activity of Ena and the WRC. In the periphery, Abl signaling in the ISNb neurons also appears to involve the same fine tuning of WRC and Ena partners interacting with 1Q. However, less is known about the specific upstream partners of $\mathrm{Abl}$ in ISNb outgrowth, but the semaphorin-plexin pathway may be a good candidate for future study. Both Semala and Plexin-A mutants display ISNb and $\mathrm{SNa}$ 
stop short phenotypes that are very similar to that of Abl $[86,87]$. Moreover, both vertebrate $\mathrm{Abl}$ and Ena have been identified as components of the Sema6A and Sema6D reverse signaling pathway [88, 89], and in Drosophila, Semala contains a putative Ena-binding site that is implicated in synaptogenesis [90]. Finally, as Sema1a signaling promotes midline crossing [91], the potential role of Drosophila Abl downstream of Semaphorinplexin signaling may provide an ideal model to understand how upstream receptors modify Abl signaling to direct a growing axon.

\section{Conclusion}

The 1Q region of Abl provides a physical link to two key components of the WRC, Hem and Sra-1, and is important in helping to regulate WRC activity during motoneuron outgrowth and midline guidance. The second half (2E) of $1 \mathrm{Q}$ and its PxxP motif are particularly important, as their removal alters the ability of Abl to function with Abi and Trio to regulate WRC activity, but surprisingly this same region is required for $\mathrm{Abl}$ to regulate Ena during axon guidance. Given that Abi and Ena interact to affect axon outgrowth of photoreceptors [76], we suggest that Abl, Abi and Ena may form an important regulatory triad, which can be tested in future work. Removal of the first half of 1Q (1E) has less distinct effects, but overall it seems this region is a likely candidate for fine tuning how Abl regulates the WRC and Ena during the formation of the different axon pathways. Together, this work extends our knowledge of how Abl participates in the regulation of both branched and linear actin dynamics, and our battery of mutants will be useful tools in future work to dissect this pathway, in particular in linking actin dynamics to upstream receptors.

\section{Supplementary information}

Supplementary information accompanies this paper at https://doi.org/10. 1186/s13064-020-00144-8.

\footnotetext{
Additional file 1 Figure $\mathbf{S} 1$. Expression levels of $A b /$ transgenes and Gal4 drivers. (A) Expression levels of $A b /$ transgenes, as assessed by western blots from 3rd instar CNS expressing transgenes with 1407-Gal4. (B) Comparison of expression levels of 1407-Gal4 and elav-Gal4 at 2 temperatures. The drivers were used to drive expression of UAS-RFP in the CNS of 3rd instar larvae. All immunoblotting was carried out against the C-terminal FLAG tag of the transgenic proteins, and are representative of three replicates.

Additional file $\mathbf{2}$ Figure S2. Abi levels perturb midline crossing over phenotypes in $\mathrm{Ab} /$ mutants. Shown here are late-stage embryonic nerve cords stained with mAb 1D4. (A) Wild-type embryo. (B) Ab/ homozygotes have occasional midline crossing overs. These are rescued by expression of (C) wild-type $A b /$ with 1407-Gal4, but not (D) $A b / \Delta 1 Q$. (E-F) Midline crossing overs are increased with $A b / \Delta 1 Q$ with heterozygous loss of $A b i$. $(\mathrm{G}-\mathrm{H})$ Overexpression of $A b i$ increases midline crossing overs, and these remain high when $A b / \Delta 1 Q$ is also expressed.
}

Additional file $\mathbf{3}$ Table S1. Mass spectrometry results from GST-1Q pulldown.

Additional file $\mathbf{4}$ Table S2. ISNb stop short counts in Abl mutants expressing Ab/ transgenes, with perturbation of WRC-related genes. Transgenes are expressed with 1407-Gal4 in conjunction with heterozygous loss of Hem, gain of Sra-1, loss or gain of Abi, or gain of trio (UAStrio. B).

Additional file $\mathbf{5}$ Table S3. ISNb stop short counts in Ab/ mutants expressing $A b /$ transgenes, with heterozygous loss of Sra-1 and trio, or gain of trio. Transgenes are expressed with 1407-Gal4 in conjunction with heterozygous loss of Hem or Sra-1. For Abl ${ }^{4 / 4}$ embryos, transgenes are expressed with elav-Gal4 with gain of trio. ${ }^{*}$ The UAS-trio (UAS-trio122) transgene used here is previously published in Kannan et al., 2017. Note that the direction of the crosses in this table is reversed compared to Table $\mathbf{S 2}$ due to a balancer-induced maternal effect (see materials \& methods).

Additional file 6 Table S4. Midline crossing over counts in Ab/ mutants expressing Ab/ transgenes, with perturbation of WRC-related genes. Transgenes are expressed with 1407-Gal4 in conjunction with heterozygous loss of Hem, gain of Sra-1, loss or gain of Abi, or gain of trio (UAS-trio.B).

Additional file $\mathbf{7}$ Table S5. Midline crossing over counts in Ab/ mutants expressing Ab/ transgenes, with perturbation of WRC-related genes. Transgenes are expressed with 1407-Gal4 in conjunction with heterozygous loss of Hem or Sra-1. Note that the direction of the crosses in this table is reversed compared to Table $\mathbf{S} 2$ due to a balancer-induced maternal effect (see methods).

Additional file $\mathbf{8}$ Table S6. Midline crossing over counts in Ab/ mutants expressing $A b /$ transgenes with elav-Gal4, with heterozygous loss and overexpression of ena.

\section{Abbreviations}

Abl: Abelson tyrosine kinase; WRC: WAVE regulatory complex; Sra1: Specifically Rac1-associated protein 1; Abi: Abelson-interacting protein; ena: Enabled; GEF: Guanine exchange factor; CTD: C-terminal domain; 1Q: First quarter; 1E: First eighth; 2E: Second eighth; P: PxxP motif; ISNb: Intersegmental nerve b; SNa: Segmental nerve a

\section{Acknowledgements}

We acknowledge the assistance of the Wayne State University Proteomics Core that is supported through NIH grants P30 ES020957, P30 CA 022453 and S10 OD010700. We appreciate the work of undergraduate students who assisted in lining up embryos for analysis, including Joseph Audisho and Ziad Khalifa. We thank Dr. Edward Giniger for discussion about Abl signaling, as well as contribution of fly stocks. The 1D4 monoclonal antibody developed by Dr. Corey Goodman, and E7 monoclonal antibody developed by Dr. Michael Klymkowsky, were obtained from the Developmental Studies Hybridoma Bank, created by the NICHD of the NIH and maintained at The University of lowa, Department of Biology, lowa City, IA 52242. Stocks obtained from the Bloomington Drosophila Stock Center (NIH P40OD018537) were used in this study.

\section{Authors' contributions}

HSJC: Conceptualization, Investigation, Formal analysis, Validation, Data Curation, Writing - Original Draft, Writing - Review \& Editing. MN: Investigation. SBG: Investigation. MVB: Conceptualization, Project administration, Supervision, Writing - Original Draft, Writing - Review \& Editing, Funding acquisition. The author(s) read and approved the final manuscript.

\section{Funding}

This research was supported by a grant from the National Science Foundation (IOS 1121025) to MVB. The funding body had no role in the study design, data collection, analysis, and interpretation, as well as writing.

\section{Availability of data and materials}

Mass spectrometry data for GST-1Q pulldowns are available in the Mendeley Data repository, at https://data.mendeley.com/datasets/mw478mgmzs/1 (DOl: https://doi.org/10.17632/mw478mgmzs.1). Other data generated or 
analysed during this study are included in this published article and its supplementary information files.

\section{Ethics approval and consent to participate}

Not applicable.

\section{Consent for publication}

Not applicable.

\section{Competing interests}

The authors declare that they have no competing interests.

Received: 14 February 2020 Accepted: 17 April 2020

Published online: 02 May 2020

\section{References}

1. Colicelli J. ABL tyrosine kinases: evolution of function, regulation, and specificity. Sci Signal. 2010;3(139):re6. PubMed PMID: 20841568. Pubmed Central PMCID: 2954126.

2. Bradley WD, Koleske AJ. Regulation of cell migration and morphogenesis by Abl-family kinases: emerging mechanisms and physiological contexts. J Cell Sci 2009 Oct 1;122(Pt 19):3441-3454. PubMed PMID: 19759284. Pubmed Central PMCID: 2746129.

3. Kannan R, Giniger E. New perspectives on the roles of Abl tyrosine kinase in axon patterning. Fly (Austin) 2017;11(4):260-270. PubMed PMID: 28481649. Pubmed Central PMCID: 5721943

4. Wang JY. The capable ABL: what is its biological function? Mol Cell Biol 2014;34(7):1188-1197. PubMed PMID: 24421390. Pubmed Central PMCID: 3993570.

5. Rogers EM, Spracklen AJ, Bilancia CG, Sumigray KD, Allred SC, Nowotarski $\mathrm{SH}$, et al. Abelson kinase acts as a robust, multifunctional scaffold in regulating embryonic morphogenesis. Mol Biol Cell. 2016;27(16):2613-2631. PubMed PMID: WOS:000382335800010. English.

6. Grevengoed EE, Loureiro JJ, Jesse TL, Peifer M. Abelson kinase regulates epithelial morphogenesis in Drosophila. J Cell Biol 2001;155(7):1185-1198. PubMed PMID: 11756472. Pubmed Central PMCID: 2199330.

7. Hsouna A, Kim YS, VanBerkum MF. Abelson tyrosine kinase is required to transduce midline repulsive cues. J Neurobiol 2003;57(1):15-30. PubMed PMID: 12973825

8. Forsthoefel DJ, Liebl EC, Kolodziej PA, Seeger MA. The Abelson tyrosine kinase, the trio GEF and enabled interact with the Netrin receptor frazzled in Drosophila. Development. 2005 Apr;132(8):1983-1994. PubMed PMID: 15790972

9. Liebl EC, Rowe RG, Forsthoefel DJ, Stammler AL, Schmidt ER, Turski M, et al.. Interactions between the secreted protein Amalgam, its transmembrane receptor Neurotactin and the Abelson tyrosine kinase affect axon pathfinding. Development. 2003;130(14):3217-3226. PubMed PMID: WOS: 000184415000013. English.

10. Wills Z, Emerson M, Rusch J, Bikoff J, Baum B, Perrimon N, et al. A Drosophila homolog of cyclase-associated proteins collaborates with the Abl tyrosine kinase to control midline axon pathfinding. Neuron. 2002;36(4): 611-622. PubMed PMID: 12441051.

11. O'Donnell MP, Bashaw GJ. Distinct functional domains of the Abelson tyrosine kinase control axon guidance responses to Netrin and slit to regulate the assembly of neural circuits. Development. 2013 Jul;140(13): 2724-2733. PubMed PMID: 23720041. Pubmed Central PMCID: 3678342.

12. Dorsten JN, Varughese BE, Karmo S, Seeger MA, VanBerkum MF. In the absence of frazzled over-expression of Abelson tyrosine kinase disrupts commissure formation and causes axons to leave the embryonic CNS. PLoS One 2010;5(3):e9822. PubMed PMID: 20352105. Pubmed Central PMCID: 2843715.

13. Bashaw GJ, Kidd T, Murray D, Pawson T, Goodman CS. Repulsive axon guidance: Abelson and enabled play opposing roles downstream of the roundabout receptor. Cell. 2000;101(7):703-715. PubMed PMID: 10892742.

14. Lee H, Engel U, Rusch J, Scherrer S, Sheard K, Van Vactor D. The microtubule plus end tracking protein orbit/MAST/CLASP acts downstream of the tyrosine kinase Abl in mediating axon guidance. Neuron. 2004;42(6):913926. PubMed PMID: 15207236.

15. Sterne GR, Kim JH, Ye B. Dysregulated Dscam levels act through Abelson tyrosine kinase to enlarge presynaptic arbors. Elife. 2015;4. PubMed PMID: WOS:000374263000001. English.
16. Wills Z, Marr L, Zinn K, Goodman CS, Van Vactor D. Profilin and the Abl tyrosine kinase are required for motor axon outgrowth in the Drosophila embryo. Neuron. 1999 Feb;22(2):291-299. PubMed PMID: 10069335.

17. Song JK, Kannan R, Merdes G, Singh J, Mlodzik M, Giniger E. Disabled is a bona fide component of the Abl signaling network. Development. 2010; 137(21):3719-3727. PubMed PMID: WOS:000283669300019. English.

18. Gertler FB, Doctor JS, Hoffmann FM. Genetic suppression of mutations in the Drosophila abl proto-oncogene homolog. Science. 1990;248(4957):857860. PubMed PMID: 2188361

19. Gertler FB, Comer AR, Juang JL, Ahern SM, Clark MJ, Liebl EC, et al.. Enabled, a Dosage-Sensitive Suppressor of Mutations in the Drosophila Abl Tyrosine Kinase, Encodes an Abl Substrate with Sh3 Domain-Binding Properties. Gene Dev. 1995;9(5):521-533. PubMed PMID: WOS:A1995QN63300002. English.

20. Lin TY, Huang $\mathrm{CH}$, Kao HH, Liou GG, Yeh SR, Cheng CM, et al. Abi plays an opposing role to $\mathrm{Abl}$ in Drosophila axonogenesis and synaptogenesis. Development. 2009;136(18):3099-3107. PubMed PMID: 19675132.

21. Kannan R, Song JK, Karpova T, Clarke A, Shivalkar M, Wang B, et al. The Abl pathway bifurcates to balance enabled and Rac signaling in axon patterning in Drosophila. Development. 2017:144(3):487-498. PubMed PMID: 28087633. Pubmed Central PMCID: 5341800

22. Comer AR, Ahern-Djamali SM, Juang JL, Jackson PD, Hoffmann FM. Phosphorylation of enabled by the Drosophila Abelson tyrosine kinase regulates the in vivo function and protein-protein interactions of enabled. Mol Cell Biol. 1998;18(1):152-160. PubMed PMID: WOS:000071195700017. English.

23. Juang $J$, Hoffmann FM. Drosophila Abelson interacting protein (dAbi) is a positive regulator of Abelson tyrosine kinase activity. Oncogene. 1999; 18(37):5138-5147. PubMed PMID: WOS:000082555700002. English

24. Henkemeyer M, West SR, Gertler FB, Hoffmann FM. A novel tyrosine kinaseindependent function of Drosophila abl correlates with proper subcellular localization. Cell. 1990;63(5):949-960. PubMed PMID: 2175256

25. Niebuhr K, Ebel F, Frank R, Reinhard M, Domann E, Carl UD, et al. A novel proline-rich motif present in ActA of listeria monocytogenes and cytoskeletal proteins is the ligand for the EVH1 domain, a protein module present in the EnaNASP family. EMBO J 1997;16(17):5433-5444. PubMed PMID: 9312002 . Pubmed Central PMCID: 1170174

26. Cheong HSJ, VanBerkum MFA. Long disordered regions of the C-terminal domain of Abelson tyrosine kinase have specific and additive functions in regulation and axon localization. PloS One. 2017;12(12). PubMed PMID: WOS:000417698200031. English.

27. Oldfield CJ, Dunker AK. Intrinsically Disordered Proteins and Intrinsically Disordered Protein Regions. Annu Rev Biochem. 2014;83:553-584. PubMed PMID: WOS:000348432500022. English.

28. Wright PE, Dyson HJ. Intrinsically disordered proteins in cellular signalling and regulation. Nat Rev Mol Cell Biol 2015;16(1):18-29. PubMed PMID: 25531225. Pubmed Central PMCID: 4405151

29. Brown CJ, Johnson AK, Dunker AK, Daughdrill GW. Evolution and disorder. Curr Opin Struct Biol 2011;21(3):441-446. PubMed PMID: 21482101. Pubmed Central PMCID: 3112239

30. Brown CJ, Takayama S, Campen AM, Vise P, Marshall TW, Oldfield CJ, et al. Evolutionary rate heterogeneity in proteins with long disordered regions. J Mol Evol 2002;55(1):104-110. PubMed PMID: 12165847.

31. Kurochkina N, Guha U. SH3 domains: modules of protein-protein interactions. Biophys Rev 2013;5(1):29-39. PubMed PMID: 28510178. Pubmed Central PMCID: 5418429.

32. Li SSC. Specificity and versatility of $\mathrm{SH} 3$ and other proline-recognition domains: structural basis and implications for cellular signal transduction. Biochem J. 2005;390:641-653. PubMed PMID: WOS:000232350200001. English.

33. Ren RB, Ye ZS, Baltimore D. Abl Protein-Tyrosine Kinase Selects the Crk Adapter as a Substrate Using Sh3-Binding Sites. Gene Dev. 1994;8(7):783795. PubMed PMID: WOS:A1994NG37600004. English.

34. Feller SM, Knudsen B, Hanafusa H. C-Abl Kinase Regulates the ProteinBinding Activity of C-Crk. Embo J. 1994;13(10):2341-2351. PubMed PMID: WOS:A1994NM54700012. English.

35. Riverolezcano OM, Marcilla A, Sameshima JH, Robbins KC. Wiskott-Aldrich Syndrome Protein Physically Associates with Nck through Src Homology-3 Domains. Mol Cell Biol. 1995;15(10):5725-5731. PubMed PMID: WOS: A1995RV77200056. English.

36. Dai Z, Pendergast AM. Abi-2, a novel SH3-containing protein interacts with the $\mathrm{c}-\mathrm{Abl}$ tyrosine kinase and modulates c-Abl transforming activity. Genes Dev 1995;9(21):2569-2582. PubMed PMID: 7590236. 
37. Shi Y, Alin K, Goff SP. Abl-interactor-1, a novel SH3 protein binding to the carboxy-terminal portion of the Abl protein, suppresses $\mathrm{v}$-abl transforming activity. Genes Dev 1995;9(21):2583-2597. PubMed PMID: 7590237.

38. Antoku S, Saksela K, Rivera GM, Mayer BJ. A crucial role in cell spreading for the interaction of Abl PxxP motifs with Crk and Nck adaptors. J Cell Sci. 2008;121(18):3071-3082. PubMed PMID: WOS:000258889400011. English.

39. Saksela K, Permi P. SH3 domain ligand binding: What's the consensus and where's the specificity? FEBS Lett 2012;586(17):2609-2614. PubMed PMID: 22710157.

40. Kobayashi K, Kuroda S, Fukata M, Nakamura T, Nagase T, Nomura N, et al.. P140Sra-1 (specifically Rac1-associated protein) is a novel specific target for Rac1 small GTPase. J Biol Chemistry. 1998;273(1):291-295. PubMed PMID: WOS:000071295600044. English.

41. Ismail AM, Padrick SB, Chen BY, Umetani J, Rosen MK. The WAVE regulatory complex is inhibited. Nat Struct Mol Biol. 2009;16(5):561-563. PubMed PMID: WOS:000266220700025. English.

42. Lebensohn AM, Kirschner MW. Activation of the WAVE Complex by Coincident Signals Controls Actin Assembly. Mol Cell. 2009;36(3):512-524. PubMed PMID: WOS:000272506200017. English.

43. Chen ZC, Borek D, Padrick SB, Gomez TS, Metlagel Z, Ismail AM, et al. Structure and control of the actin regulatory WAVE complex. Nature. 2010; 468(7323):533-U207. PubMed PMID: WOS:000284584200035. English.

44. Studier FW. Stable expression clones and auto-induction for protein production in E. coli. Methods Mol Biol 2014;1091:17-32. PubMed PMID: 24203322.

45. Bateman J, Shu H, Van Vactor D. The guanine nucleotide exchange factor trio mediates axonal development in the Drosophila embryo. Neuron. 2000 Apr;26(1):93-106. PubMed PMID: 10798395.

46. Bischof J, Maeda RK, Hediger M, Karch F, Basler K. An optimized transgenesis system for Drosophila using germ-line-specific phi C31 integrases. P Natl Acad Sci USA. 2007;104(9):3312-3317. PubMed PMID: WOS: 000244661400053. English.

47. Zhu Z, Bhat KM. The Drosophila hem/Kette/Nap1 protein regulates asymmetric division of neural precursor cells by regulating localization of Inscuteable and numb. Mech Dev 2011;128(7-10):483-495. PubMed PMID: 21996673. Pubmed Central PMCID: PMC3263386.

48. Campos-Ortega JA, Hartenstein V. Stages of Drosophila Embryogenesis. The Embryonic Development of Drosophila melanogaster. Berlin, Heidelberg. 1985:9-84

49. Hothorn T, Bretz F, Westfall P. Simultaneous inference in general parametric models. Biom J 2008 Jun;50(3):346-363. PubMed PMID: 18481363.

50. Venables WN, Ripley BD, Venables WN. Modern applied statistics with S. 4th ed. New York: Springer; 2002. xi, 495 p. p.

51. Wickham H.. Ggplot2 : elegant graphics for data analysis. New York: Springer; 2009. viii, 212 p. p.

52. Fox J, Weisberg S, Fox J. An R companion to applied regression. 2nd ed. Thousand Oaks, Calif.: SAGE Publications; 2011. xxii, 449 p. p.

53. Cameron AC, Trivedi PK. Regression-Based Tests for Overdispersion in the Poisson Model. J Econometrics. 1990;46(3):347-364. PubMed PMID: WOS: A1990EL35200005. English.

54. Cameron AC, Trivedi PK. Regression analysis of count data. Cambridge, UK ; New York, NY, USA: Cambridge University Press; 1998. xvii, 411 p. p.

55. Cameron AC, Trivedi PK. Microeconometrics : methods and applications. Cambridge ; New York: Cambridge University Press; 2005. xxii, 1034 p. p.

56. Searle SR, Speed FM, Milliken GA. Population Marginal Means in the LinearModel - an Alternative to Least-Squares Means. Am Stat. 1980;34(4):216-221. PubMed PMID: WOS:A1980KT98100004. English.

57. Brant R. Assessing proportionality in the proportional odds model for ordinal logistic regression. Biometrics. 1990;46(4):1171-1178. PubMed PMID: 2085632

58. Cheong HSJ, VanBerkum MF. Mass spectrometry data for pulldown of the Abelson first quarter region from Drosophila lysate. 1 ed. Mendeley Data 2019.

59. Chen Z, Borek D, Padrick SB, Gomez TS, Metlagel Z, Ismail AM, et al. Structure and control of the actin regulatory WAVE complex. Nature. 2010; 468(7323):533-538. PubMed PMID: 21107423. Pubmed Central PMCID: 3085272.

60. Weiner OD, Rentel MC, Ott A, Brown GE, Jedrychowski M, Yaffe MB, et al. Hem-1 complexes are essential for Rac activation, actin polymerization, and myosin regulation during neutrophil chemotaxis. PLoS Biol 2006 Feb;4(2): e38. PubMed PMID: 16417406. Pubmed Central PMCID: PMC1334198.

61. Campos-Ortega JA, Hartenstein V. The embryonic development of Drosophila melanogaster. 2nd ed. Berlin ; New York: Springer; 1997. xvii, 405 p. p.
62. Landgraf $M$, Thor S. Development of Drosophila motoneurons: specification and morphology. Semin Cell Dev Biol 2006;17(1):3-11. PubMed PMID: 16356739.

63. Wills Z, Bateman J, Korey CA, Comer A, Van Vactor D. The tyrosine kinase $\mathrm{Abl}$ and its substrate Enabled collaborate with the receptor phosphatase Dlar to control motor axon guidance. Neuron. 1999;22(2):301-312. PubMed PMID: WOS:000078875200015. English.

64. Schenck A, Qurashi A, Carrera P, Bardoni B, Diebold C, Schejter E, et al. WAVE/SCAR, a multifunctional complex coordinating different aspects of neuronal connectivity. Dev Biol 2004;274(2):260-270. PubMed PMID: 15385157. Epub 2004/09/24

65. Innocenti M, Zucconi A, Disanza A, Frittoli E, Areces LB, Steffen A, et al. Abi1 is essential for the formation and activation of a WAVE2 signalling complex. Nat Cell Biol. 2004;6(4):319-327. PubMed PMID: WOS:000220587600013. English.

66. Chen B, Brinkmann K, Chen Z, Pak CW, Liao Y, Shi S, et al. The WAVE regulatory complex links diverse receptors to the actin cytoskeleton. Cell. 2014;156(1-2):195-207. PubMed PMID: 24439376. Pubmed Central PMCID: PMC4059610.

67. Kobayashi K, Kuroda S, Fukata M, Nakamura T, Nagase T, Nomura N, et al. p140Sra-1 (specifically Rac1-associated protein) is a novel specific target for Rac1 small GTPase. J Biol Chem 1998;273(1):291-295. PubMed PMID: 9417078.

68. Schenck A, Bardoni B, Langmann C, Harden N, Mandel JL, Giangrande A. CYFIP/Sra-1 controls neuronal connectivity in Drosophila and links the Rac1 GTPase pathway to the fragile X protein. Neuron. 2003;38(6):887-898. PubMed PMID: 12818175.

69. Pollitt AY, Insall RH. WASP and SCAR/WAVE proteins: the drivers of actin assembly. J Cell Sci 2009;122(Pt 15):2575-2578. PubMed PMID: 19625501. Pubmed Central PMCID: PMC2954249. Epub 2009/07/25.

70. Grevengoed EE, Fox DT, Gates J, Peifer M. Balancing different types of actin polymerization at distinct sites: roles for Abelson kinase and enabled. J Cell Biol 2003;163(6):1267-1279. PubMed PMID: 14676307. Pubmed Central PMCID: 2173720

71. Kannan R, Kuzina I, Wincovitch S, Nowotarski SH, Giniger E. The Abl/enabled signaling pathway regulates Golgi architecture in Drosophila photoreceptor neurons. Mol Biol Cell 2014;25(19):2993-3005. PubMed PMID: 25103244. Pubmed Central PMCID: 4230588.

72. Grevengoed EE, Fox DT, Gates J, Peifer M. Balancing different types of actin polymerization at distinct sites: roles for Abelson kinase and Enabled. J Cell Biol. 2003;163(6):1267-1279. PubMed PMID: WOS:000187583500010. English

73. Davidson AJ, Ura S, Thomason PA, Kalna G, Insall RH. Abi is required for modulation and stability but not localization or activation of the SCAR/ WAVE complex. Eukaryot Cell. 2013;12(11):1509-16.

74. Litschko C, Linkner J, Bruhmann S, Stradal TEB, Reinl T, Jansch L, et al. Differential functions of WAVE regulatory complex subunits in the regulation of actin-driven processes. Eur J Cell Biol 2017:96(8):715-727. PubMed PMID: 28889942.

75. Chen B, Chou HT, Brautigam CA, Xing W, Yang S, Henry L, et al. Rac1 GTPase activates the WAVE regulatory complex through two distinct binding sites. Elife. 2017 26;6. PubMed PMID: 28949297. Pubmed Central PMCID: 5614565.

76. Chen XJ, Squarr AJ, Stephan R, Chen B, Higgins TE, Barry DJ, et al. EnaNASP proteins cooperate with the WAVE complex to regulate the actin cytoskeleton. Dev Cell 2014;30(5):569-584. PubMed PMID: 25203209. Pubmed Central PMCID: 4165403.

77. Krause M, Gautreau A. Steering cell migration: lamellipodium dynamics and the regulation of directional persistence. Nat Rev Mol Cell Biol 2014;15(9): 577-590. PubMed PMID: 25145849.

78. Goley ED, Welch MD. The ARP2/3 complex: an actin nucleator comes of age. Nat Rev Mol Cell Biol 2006;7(10):713-726. PubMed PMID: 16990851.

79. Bear JE, Gertler FB. EnaNASP: towards resolving a pointed controversy at the barbed end. J Cell Sci 2009;122(Pt 12):1947-1953. PubMed PMID: 19494122. Pubmed Central PMCID: 2723151.

80. Bear JE, Svitkina TM, Krause M, Schafer DA, Loureiro JJ, Strasser GA, et al. Antagonism between Ena/NASP proteins and actin filament capping regulates fibroblast motility. Cell. 2002;109(4):509-521. PubMed PMID: 12086607.

81. Clarke A, McQueen PG, Fang HY, Kannan R, Wang V, McCreedy E, et al. Abl signaling directs growth of a pioneer axon in Drosophila by shaping the intrinsic fluctuations of actin. Mol Biol Cell. 2020:mbcE19100564. PubMed PMID: 31967946 
82. Clarke A, McQueen PG, Fang HY, Kannan R, Wang V, McCreedy E, et al. Dynamic morphogenesis of a pioneer axon in Drosophila and its regulation by Abl tyrosine kinase. Mol Biol Cell. 2020:mbcE19100563. PubMed PMID: 31967935.

83. Fan XP, Labrador JP, Hing H, Bashaw GJ. Slit stimulation recruits Dock and Pak to the roundabout receptor and increases Rac activity to regulate axon repulsion at the CNS midline. Neuron. 2003;40(1):113-127. PubMed PMID: WOS:000185614700013. English.

84. Bernadskaya YY, Wallace A, Nguyen J, Mohler WA, Soto MC. UNC-40/DCC, SAX-3/Robo, and VAB-1/Eph polarize F-actin during embryonic morphogenesis by regulating the WAVE/SCAR actin nucleation complex. PLoS Genet 2012;8(8):e1002863. PubMed PMID: 22876199. Pubmed Central PMCID: 3410845

85. McConnell RE, Edward van Veen J, Vidaki M, Kwiatkowski AV, Meyer AS, Gertler FB. A requirement for filopodia extension toward slit during Robomediated axon repulsion. J Cell Biol 2016;213(2):261-274. PubMed PMID: 27091449. Pubmed Central PMCID: 5084274

86. Yu HH, Araj HH, Ralls SA, Kolodkin AL. The transmembrane semaphorin Sema I is required in Drosophila for embryonic motor and CNS axon guidance. Neuron. 1998;20(2):207-220. PubMed PMID: WOS: 000072154700007. English.

87. Winberg ML, Noordermeer JN, Tamagnone L, Comoglio PM, Spriggs MK Tessier-Lavigne $M$, et al. Plexin a is a neuronal semaphorin receptor that controls axon quidance. Cell. 1998;95(7):903-916. PubMed PMID: 9875845.

88. Toyofuku T, Zhang H, Kumanogoh A, Takegahara N, Yabuki M, Harada K, et al. Guidance of myocardial patterning in cardiac development by Sema6D reverse signalling. Nat Cell Biol 2004;6(12):1204-1211. PubMed PMID: 15543137

89. Perez-Branguli F, Zagar Y, Shanley DK, Graef IA, Chedotal A, Mitchell KJ. Reverse Signaling by Semaphorin-6A Regulates Cellular Aggregation and Neuronal Morphology. PloS One. 2016;11(7). PubMed PMID: WOS: 000380005400102. English.

90. Godenschwege TA, Hu H, Shan-Crofts X, Goodman CS, Murphey RK. Bidirectional signaling by Semaphorin 1a during central synapse formation in Drosophila. Nat Neurosci 2002;5(12):1294-1301. PubMed PMID: 12436113.

91. Hernandez-Fleming M, Rohrbach EW, Bashaw GJ. Sema-1a reverse signaling promotes midline crossing in response to secreted Semaphorins. Cell Rep 2017;18(1):174-184. PubMed PMID: 28052247. Pubmed Central PMCID: 5253228.

\section{Publisher's Note}

Springer Nature remains neutral with regard to jurisdictional claims in published maps and institutional affiliations.

Ready to submit your research? Choose BMC and benefit from:

- fast, convenient online submission

- thorough peer review by experienced researchers in your field

- rapid publication on acceptance

- support for research data, including large and complex data types

- gold Open Access which fosters wider collaboration and increased citations

- maximum visibility for your research: over $100 \mathrm{M}$ website views per year

At $\mathrm{BMC}$, research is always in progress.

Learn more biomedcentral.com/submissions 